\title{
STRUCTURED EIGENVALUE BACKWARD ERRORS OF MATRIX PENCILS AND POLYNOMIALS WITH PALINDROMIC STRUCTURES*
}

\author{
SHREEMAYEE BORA ${ }^{\dagger}$, MICHAEL KAROW $^{\ddagger}$, CHRISTIAN MEHL $^{\dagger}$, \\ AND PUNIT SHARMA $\ddagger$
}

\begin{abstract}
We derive formulas for the backward error of an approximate eigenvalue of a $*-$ palindromic matrix polynomial with respect to $*$-palindromic perturbations. Such formulas are also obtained for complex $T$-palindromic pencils and quadratic polynomials. When the $T$-palindromic polynomial is real, then we derive the backward error of a real number considered as an approximate eigenvalue of the matrix polynomial with respect to real $T$-palindromic perturbations. In all cases the corresponding minimal structure preserving perturbations are obtained as well. The results are illustrated by numerical experiments. These show that there is a significant difference between the backward errors with respect to structure preserving and arbitrary perturbations in many cases.
\end{abstract}

Key words. palindromic matrix pencil, palindromic matrix polynomial, perturbation theory, eigenvalue backward error, structured eigenvalue backward error

AMS subject classifications. 15A22, 15A18, 47A56, 15A60, 65F15, 65F30, 93C73

DOI. $10.1137 / 140973839$

1. Introduction. Given $n \times n$ matrices $A_{0}, \ldots, A_{m}$, the corresponding matrix polynomial $P(z):=A_{0}+z A_{1}+\cdots+z^{m} A_{m}$ is said to have an eigenvalue $\lambda \in \mathbb{C}$ with corresponding eigenvector $v \in \mathbb{C}^{n} \backslash\{0\}$ if $P(\lambda) v=0$. We consider such eigenvalue problems for the special case that the coefficient matrices of $P(z)$ satisfy certain symmetries. This is indicated by stating that the ordered tuple $\left(A_{0}, \ldots, A_{m}\right)$ belongs to $\mathbb{S}$, where $\mathbb{S} \subset\left(\mathbb{C}^{n \times n}\right)^{m+1}$. In particular, given $\lambda \in \mathbb{C}$, we are interested in perturbations $\left(\Delta_{0}, \ldots, \Delta_{m}\right) \in \mathbb{S}$ to $\left(A_{0}, \ldots, A_{m}\right) \in \mathbb{S}$ that are minimal with respect to a specified norm such that $\lambda$ is an eigenvalue of the perturbed polynomial $\widetilde{P}(z):=\left(A_{0}-\Delta_{0}\right)+z\left(A_{1}-\Delta_{1}\right)+\cdots+z^{m}\left(A_{m}-\Delta_{m}\right)$. The norm of such a minimal structure preserving perturbation is called the structured backward error of $\lambda$ as an approximate eigenvalue of $P(z)$. We refer to this also as the structured eigenvalue backward error of $\lambda$ with respect to $P(z)$ and $\mathbb{S}$. In contrast, we refer to the norm of a minimal but not necessarily structure preserving perturbation to $P(z)$ such that $\lambda \in \mathbb{C}$ is an eigenvalue of the perturbed polynomial simply as the eigenvalue backward error of $\lambda$ with respect to $P(z)$.

Matrix polynomials with symmetries in their coefficients are referred to as structured matrix polynomials. For example, the coefficients of Hermitian matrix polynomials are all Hermitian matrices, i.e., they satisfy, $A_{j}^{*}=A_{j}$, for $j=0,1, \ldots, m$, where $A^{*}$ denotes the complex conjugate transpose of a matrix $A$. Other structured matrix polynomials closely related to the Hermitian matrix polynomials are the skewHermitian matrix polynomials where the coefficients are all skew-Hermitian matrices

*Received by the editors June 19, 2014; accepted for publication (in revised form) by F. Tisseur January 28, 2015; published electronically April 14, 2015. This research was supported by DAAD and DST through the project Distance Problems for Structured Matrix Polynomials within the Personal Exchange Programme PPP.

http://www.siam.org/journals/simax/36-2/97383.html

${ }^{\dagger}$ Department of Mathematics, Indian Institute of Technology Guwahati, Guwahati-781039, India (shbora@iitg.ernet.in, s.punit@iitg.ernet.in).

${ }^{\ddagger}$ Institut für Mathematik, Technische Universität Berlin, 10623 Berlin, Germany (karow@math.tu-berlin.de, mehl@math.tu-berlin.de). 
and $*$-even and $*$-odd matrix polynomials where the coefficient matrices alternate between Hermitian and skew-Hermitian structure, due to which they are also referred to as *-alternating polynomials [22]. Yet another interesting class of structured matrix polynomials is the $*$-palindromic polynomials where the coefficient matrices satisfy $A_{j}^{*}=A_{m-j}$ for $j=0, \ldots, m$. The $*$-palindromic polynomials $P(z)$ have the property that $\operatorname{rev} P^{*}(z)=P(z)$, where $P^{*}(z):=\sum_{j=0}^{m} z^{j} A_{j}^{*}$, and $\operatorname{rev} P(z):=z^{m} P(1 / z)$ represents the reversal of $P(z)$ obtained by reversing the order of the coefficient matrices in $P(z)$. Replacing the complex conjugate transpose $*$ by the transpose $T$ in the above definition of $*$-palindromic matrix polynomials results in the $T$-palindromic matrix polynomials which satisfy $\operatorname{rev} P^{T}(z)=P(z)$. Each of the above mentioned structured matrix polynomials displays a symmetry in its eigenvalue distribution as an immediate consequence of the symmetry in the coefficient matrices. For example, the eigenvalues of Hermitian and skew-Hermitian matrix polynomials occur in pairs $(\lambda, \bar{\lambda})$. Eigenvalues of $*$-even and $*$-odd matrix polynomials occur in pairs $(\lambda,-\bar{\lambda})$ when the polynomial is complex and in quadruples $(\lambda,-\bar{\lambda}, \bar{\lambda},-\lambda)$ when the polynomial is real. On the other hand, for complex $*$-palindromic and $T$-palindromic matrix polynomials, these pairings are $(\lambda, 1 / \bar{\lambda})$ and $(\lambda, 1 / \lambda)$, respectively. If these matrix polynomials are real, then the eigenvalues occur in quadruples $(\lambda, 1 / \bar{\lambda}, \bar{\lambda}, 1 / \lambda)$.

This paper is a follow-up to [6], which considered the similar problem of finding structured backward errors of approximate eigenvalues and associated minimal structure preserving perturbations for the particular case of Hermitian matrix polynomials and related structures like skew-Hermitian and $*$-alternating matrix polynomials. In the same work, it was stated that when the problem concerns $*$-palindromic and $T$ palindromic structures, the solutions require a different treatment from that of the Hermitian and related structures, and the aim of this work is to focus on such structures.

As mentioned in [6], structured matrix polynomials occur widely in various applications. For example, Hermitian matrix polynomials occur in structural mechanics, fluid flows, and signal processing, to name a few (for details, see [28] and references therein), while $*$-even matrix polynomials arise in linear quadratic optimal control problems $[21,25]$ and in gyroscopic systems [20]. On the other hand, *-palindromic matrix polynomials occur in discrete time optimal control theory [22] and $T$-palindromic matrix polynomials arise in the mathematical modeling and numerical simulation of surface acoustic wave filters [29] and in the vibration analysis of railway tracks excited by high-speed trains $[12,13]$. It is well established [28] that it is important to use algorithms that preserve the structure of the matrix polynomials when computing their eigenvalues so that the eigenvalue pairing associated with the structure remains intact under round-off errors and any analysis or application based on them is physically meaningful and useful. Therefore, structure preserving perturbation and backward error analysis of eigenvalues and eigenvectors of structured matrix polynomials play a crucial role in all applications involving such polynomials. Significant contributions toward structured backward error and perturbation analysis have been made in $[2,3,4,10,16,18,27]$.

The problem of finding minimal structure preserving perturbations to a given structured polynomial $P(z)$ such that the perturbed polynomial has a prescribed eigenvalue $\lambda$ and a corresponding eigenvector $v$ gives the structured backward error for the approximate eigenpair $(\lambda, v)$ of $P(z)$. This problem has been solved for several classes of structured matrix pencils and polynomials in $[2,3]$. However, except for the work done in [6], the problem of computing structured eigenvalue backward errors has not been undertaken so far. 
The structured eigenvalue backward errors are important for the stability analysis of structure preserving algorithms like those in $[19,23]$ that compute only eigenvalues. They are also important for finding solutions to distance problems involving structured matrices as in the case of the distance to bounded realness for Hamiltonian matrices [5] and in the passivation of linear time invariant control systems. The backward error for an approximate eigenvalue $\lambda$ of $P(z)$ may be obtained by minimizing the corresponding expression for the backward error of the approximate eigenpair $(\lambda, v)$ over all nonzero vectors $v$. But, the expressions for the structured backward errors for approximate eigenpairs in $[2,3]$ indicate that this approach may not be feasible for structured eigenvalue backward errors. There are certain special situations when the backward errors of approximate eigenvalues are equal with respect to structured and arbitrary perturbations. For instance, this is the case for approximate real eigenvalues of Hermitian and skew-Hermitian matrix polynomials, purely imaginary approximate eigenvalues of $*$-alternating matrix polynomials, and approximate eigenvalues on the unit circle of $*$-palindromic matrix polynomials $[1,2,3]$. Apart from these cases, in all other situations, the computation of the structured eigenvalue backward error is a challenging and important problem.

This paper is organized as follows. In section 2, we formulate our problems, provide definitions, and give the preliminary results necessary for the computation of the structured eigenvalue backward errors. In section 3, we reformulate the original problem of computing the structured eigenvalue backward error as an equivalent problem of maximizing the Rayleigh quotient of a Hermitian matrix with respect to certain constraints. We derive formulas for the structured eigenvalue backward error of $\lambda \in \mathbb{C}$ for $*$-palindromic polynomials in section 4 and the $T$-palindromic pencils and quadratic polynomials in section 5 . We also find formulas for the structured backward error of $\lambda \in \mathbb{R}$ for real $T$-palindromic polynomials of any degree with respect to real $T$-palindromic perturbations.

Notation. We use the notation $\operatorname{Herm}(n)$ and $\operatorname{Sym}(n)$ to denote the sets of Hermitian and symmetric matrices of size $n \times n$, respectively. The notations $\lambda_{\max }(H)$ and $\lambda_{2}(H)$ denote the largest and second largest eigenvalues of a Hermitian matrix $H$, respectively. Also, $\sigma_{2}(S)$ denotes the second largest singular value of a matrix $S$. The symbol $\otimes$ denotes the Kronecker product of matrices (or vectors) and $\|\cdot\|$ refers to the spectral norm.

2. Preliminaries. We introduce a norm on $\left(\mathbb{C}^{n \times n}\right)^{m+1}$ associated with a weight vector $w \in \mathbb{R}^{m+1}$ to be able to measure perturbations of matrix polynomials in a flexible way.

Definition 2.1. Let $w=\left(w_{0}, \ldots, w_{m}\right) \in \mathbb{R}^{m+1}$, where $w_{0}, \ldots, w_{m}>0$.

(1) $w$ is called $a$ weight vector and its entries $w_{j}$ are called weights.

(2) The reciprocal weight vector of $w$ is defined as $w^{-1}:=\left(w_{0}^{-1}, \ldots, w_{m}^{-1}\right)$.

(3) A weight vector $w=\left(w_{0}, \ldots, w_{m}\right)$ is said to be a palindromic weight vector if $w_{j}=w_{m-j}$ for $j=0, \ldots, m$.

(4) For a tuple of matrices $\Delta_{0}, \ldots, \Delta_{m} \in \mathbb{C}^{n \times n}$, we define

$$
\left\|\left(\Delta_{0}, \ldots, \Delta_{m}\right)\right\|_{w}:=\sqrt{w_{0}^{2}\left\|\Delta_{0}\right\|^{2}+\cdots+w_{m}^{2}\left\|\Delta_{m}\right\|^{2}} .
$$

Definition 2.2. Let $P(z)=z^{m} A_{m}+\cdots+z A_{1}+A_{0}$ be a matrix polynomial, where $A_{0}, \ldots, A_{m} \in \mathbb{C}^{n \times n}$, and let $\lambda \in \mathbb{C}$. Furthermore, let $w=\left(w_{0}, \ldots, w_{m}\right) \in \mathbb{R}^{m+1}$ be a weight vector and let $\mathbb{S} \subseteq\left(\mathbb{C}^{n \times n}\right)^{m+1}$. Then we call

$$
\eta_{w}^{\mathbb{S}}(P, \lambda):=\inf \left\{\left\|\left(\Delta_{0}, \ldots, \Delta_{m}\right)\right\|_{w} \mid \operatorname{det}\left(\sum_{j=0}^{m} \lambda^{j}\left(A_{j}-\Delta_{j}\right)\right)=0,\left(\Delta_{0}, \ldots, \Delta_{m}\right) \in \mathbb{S}\right\}
$$


the backward error of $\lambda$ with respect to $P(z), \mathbb{S}$, and $w$.

Thus, $\eta_{w}^{\mathbb{S}}(P, \lambda)$ is the norm of the smallest perturbation from $\mathbb{S}$ so that $\lambda$ becomes an eigenvalue of the perturbed matrix polynomial $\widetilde{P}(z)=\sum_{j=0}^{m} z^{j}\left(A_{j}-\Delta_{j}\right)$. Clearly, we have $\eta_{w}^{\mathbb{S}}(P, \lambda)=0$ if the matrix $P(\lambda) \in \mathbb{C}^{n \times n}$ is singular, i.e., if $\lambda$ is already an eigenvalue of $P(z)$ (including the case that the matrix polynomial $P(z)$ is singular). Throughout this paper we assume that $P(z)$ is regular and that $P(\lambda)$ is nonsingular. Also it is important to note that if $\left(A_{0}, \ldots, A_{m}\right) \in \mathbb{S}$, then

$$
\eta_{w}^{\mathbb{S}}(P, \lambda) \leq\left\|\left(A_{0}, \ldots, A_{m}\right)\right\|_{w}<\infty,
$$

because the perturbation with the tuple $\left(A_{0}, \ldots, A_{m}\right)$ results in the zero polynomial. The case $\mathbb{S}=\mathbb{C}^{n \times n}$ in the definition of $\eta_{w}^{\mathbb{S}}(P, \lambda)$ corresponds to the backward error with respect to arbitrary perturbations and we denote this by $\eta_{w}(P, \lambda)$. It is well known (see, for example, [4, Proposition 4.6] or [26, Theorem 1]) that

$$
\eta_{w}(P, \lambda)=\frac{\sigma_{\min }(P(\lambda))}{\left\|\left(1, \lambda, \ldots, \lambda^{m}\right)\right\|_{w^{-1}}},
$$

where $\sigma_{\min }(A)$ stands for the smallest singular value of a matrix $A$.

For brevity, whenever we make statements that are valid for both $*$-palindromic and $T$-palindromic structures, we use the term $\star$-palindromic, where $\star=*$ or $\star=T$. Thus, denoting the $\star$-palindromic structure by $\mathrm{pal}_{\star}$, we have

$$
\operatorname{pal}_{\star}= \begin{cases}\left\{\left(A_{0}, \ldots, A_{m}\right) \in\left(\mathbb{C}^{n \times n}\right)^{m+1}: A_{j}^{*}=A_{m-j}\right\} & \text { if } \star=*, \\ \left\{\left(A_{0}, \ldots, A_{m}\right) \in\left(\mathbb{C}^{n \times n}\right)^{m+1}: A_{j}^{T}=A_{m-j}\right\} & \text { if } \star=T .\end{cases}
$$

The flexibility to perturb a polynomial with coefficients in pal $_{\star}$ in a structure preserving way is restricted by the fact that equal weights must be given to coefficients in position $j$ and position $m-j$. Therefore unless otherwise stated, we assume that the weight vector $w$ is a palindromic weight vector as defined in Definition 2.1. Our aim will be to solve the following problem.

Problem 2.3. Let $P(z)=\sum_{i=0}^{m} z^{j} A_{j}$ be $\star$-palindromic and $\lambda \in \mathbb{C} \backslash\{0\}$. Suppose that $P(\lambda)$ is nonsingular. Find the smallest structured perturbation from pal $\mathrm{l}_{\star}$ that makes $\lambda$ an eigenvalue of the perturbed $\star$-palindromic polynomial. More precisely, calculate

$\eta_{w}^{\mathrm{pal}_{\star}}(P, \lambda):=\inf \left\{\left\|\left(\Delta_{0}, \ldots, \Delta_{m}\right)\right\|_{w} \mid \operatorname{det}\left(\sum_{j=0}^{m} \lambda^{j}\left(A_{j}-\Delta_{j}\right)\right)=0,\left(\Delta_{0}, \ldots, \Delta_{m}\right) \in \operatorname{pal}_{\star}\right\}$

and construct the corresponding perturbation $\Delta P(z)=\sum_{j=0}^{m} \lambda^{j} \Delta_{j}$ that attains the infimum.

Note that the assumption $\lambda \in \mathbb{C} \backslash\{0\}$ is justified because

$$
\eta_{w}^{\mathrm{pal}_{\star}}(P, 0)=\sqrt{2} w_{0} \sigma_{\min }\left(A_{0}\right),
$$

where $\sigma_{\min }\left(A_{0}\right)$ is the minimum singular value of $A_{0}$. Also note that by restricting all the entries of the weight vector to be positive in Definition 2.1, we are allowing only those perturbations to $P(z)$ that affect all its coefficient matrices. We consider perturbations that leave certain coefficient matrices of $P(z)$ unchanged to be elements of some subset of $\left(\mathbb{C}^{n \times n}\right)^{p}$, where $p<m$ is a positive integer determined by the number of coefficient matrices of $P(z)$ that are perturbed. The backward error $\eta_{w}^{\text {pal }_{\star}}(P, \lambda)$ can 
be computed with respect to such perturbations along the lines of [6, section 6$]$, where this is done for Hermitian matrix polynomials. Brief discussions on the corresponding strategies for the $*$-palindromic and $T$-palindromic matrix polynomials are provided in Remarks 4.4 and 5.5, respectively.

We follow the strategy used in [6] to reformulate the problem of computing $\eta_{w}^{\mathbb{S}}(P, \lambda)$ in terms of a structured mapping problem. A key result in this respect is [6, Lemma 2.4]. It states that if any $\lambda \in \mathbb{C}$ is not an eigenvalue of an $n \times n$ matrix polynomial $P(z)=\sum_{j=0}^{m} z^{j} A_{j}$, then $\lambda$ is an eigenvalue of a perturbed polynomial $\tilde{P}(z):=\sum_{j=0}^{m} z^{j}\left(A_{j}-\Delta_{j}\right)$ if and only if there exist vectors $v_{0}, \ldots, v_{m} \in \mathbb{C}^{n}$ satisfying $\sum_{j=0}^{m} \lambda^{j} v_{j} \neq 0$ such that

$$
v_{j}=\Delta_{j} M\left(\lambda^{m} v_{m}+\cdots+\lambda v_{1}+v_{0}\right) \text { for } j=0, \ldots, m,
$$

where $M:=(P(\lambda))^{-1}$. This result yields the following alternative characterization of $\eta_{w}^{\mathrm{pal}_{*}}(P, \lambda)$ in terms of mapping problems.

Lemma 2.4. Let $P(z)=\sum_{j=0}^{m} z^{j} A_{j}$ be $\star$-palindromic and $\lambda \in \mathbb{C} \backslash\{0\}$. Also let $k:=\left\lfloor\frac{m-1}{2}\right\rfloor$ and $v_{\lambda}:=\sum_{j=0}^{m} \lambda^{j} v_{j}$, where $v_{0}, \ldots, v_{m} \in \mathbb{C}^{n}$. Assume that $P(\lambda)$ is nonsingular and let $M=(P(\lambda))^{-1}$. If $m$ is odd,

$$
\begin{gathered}
\eta_{w}^{\mathrm{pal}_{\star}}(P, \lambda)=\inf \left\{\left\|\left(\Delta_{0}, \ldots, \Delta_{m}\right)\right\|_{w} \mid \exists v_{0}, \ldots, v_{m} \in \mathbb{C}^{n}, v_{\lambda} \neq 0,\left(\Delta_{0}, \ldots, \Delta_{m}\right) \in \mathrm{pal}_{\star},\right. \\
\left.\Delta_{j} M v_{\lambda}=v_{j}, \Delta_{j}^{\star} M v_{\lambda}=v_{m-j}, \quad j=0, \ldots, k\right\},
\end{gathered}
$$

and if $m$ is even,

$$
\begin{gathered}
\eta_{w}^{\mathrm{pal}_{\star}}(P, \lambda)=\inf \left\{\left\|\left(\Delta_{0}, \ldots, \Delta_{m}\right)\right\|_{w} \mid \exists v_{0}, \ldots, v_{m} \in \mathbb{C}^{n}, v_{\lambda} \neq 0,\left(\Delta_{0}, \ldots, \Delta_{m}\right) \in \mathrm{pal}_{\star},\right. \\
\left.\Delta_{\frac{m}{2}} M v_{\lambda}=v_{\frac{m}{2}}, \Delta_{j} M v_{\lambda}=v_{j}, \Delta_{j}^{\star} M v_{\lambda}=v_{m-j}, \quad j=0, \ldots, k\right\} .
\end{gathered}
$$

Necessary and sufficient conditions for the mapping problems

$$
\Delta_{j} M v_{\lambda}=v_{j}, \Delta_{j}^{\star} M v_{\lambda}=v_{m-j}
$$

in Lemma 2.4 to be solvable as well as minimal norm solutions to such problems have been obtained in [14, Theorem 2]. We restate the result with an alternative proof and include a formula for the desired minimal norm solution.

Theorem 2.5. Let $x, y, z \in \mathbb{C}^{n}$ with $x \neq 0$. Then there exists a matrix $\Delta \in \mathbb{C}^{n \times n}$ such that $\Delta x=y$ and $\Delta^{\star} x=z$ if and only if $x^{\star} y=z^{\star} x$. If the latter condition is satisfied, then

$$
\min \left\{\|\Delta\| \mid \Delta \in \mathbb{C}^{n \times n}, \Delta x=y, \Delta^{\star} x=z\right\}=\max \left\{\frac{\|y\|}{\|x\|}, \frac{\|z\|}{\|x\|}\right\} .
$$

Furthermore, let $\hat{x}=x$ when $\star=*$ and $\hat{x}=\bar{x}$ when $\star=T$, and let $y_{1}$ and $z_{1}$ denote the orthogonal projections of $y$ and $z$, respectively, onto the orthogonal complement of $\hat{x}$. If $\left\|z_{1}\right\| \leq\left\|y_{1}\right\|$, then the minimum in (2.1) is attained for

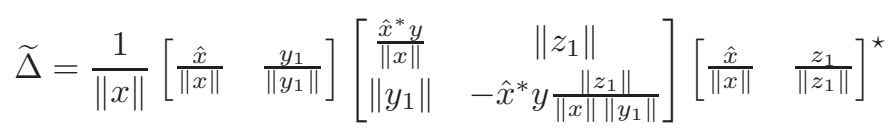

Copyright $@$ by SIAM. Unauthorized reproduction of this article is prohibited. 
if $z_{1} \neq 0$ and for $\widetilde{\Delta}=\frac{1}{\|x\|^{2}} y x^{*}$ if $z_{1}=0$. If $\left\|y_{1}\right\| \leq\left\|z_{1}\right\|$, then these formulas can be used to construct $\widetilde{\Delta}^{\star}$.

Proof. Clearly, the identities $\Delta x=y$ and $\Delta^{\star} x=z$ imply $x^{\star} y=z^{\star} x$ and

$$
\|\Delta\| \geq \max \left\{\frac{\|y\|}{\|x\|}, \frac{\|z\|}{\|x\|}\right\} .
$$

Suppose now that $x^{\star} y=z^{\star} x$ holds true. Denote $x_{0}=\hat{x} /\|x\|$. Then

$$
\begin{aligned}
& y=\left(x_{0}^{*} y\right) x_{0}+y_{1}, x_{0}^{*} y_{1}=0,\|y\|^{2}=\left|x_{0}^{*} y\right|^{2}+\left\|y_{1}\right\|^{2}, \\
& z=\left(x_{0}^{*} z\right) x_{0}+z_{1}, x_{0}^{*} z_{1}=0,\|z\|^{2}=\left|x_{0}^{*} z\right|^{2}+\left\|z_{1}\right\|^{2} .
\end{aligned}
$$

Notice that $x_{0}^{*} y=z^{*} x_{0}$ and hence $\|y\|^{2}-\|z\|^{2}=\left\|y_{1}\right\|^{2}-\left\|z_{1}\right\|^{2}$. For every $\alpha \in \mathbb{C}$ the matrix

$$
\Delta_{\alpha}=\|x\|^{-1}\left(\left(x_{0}^{*} y\right) x_{0} x_{0}^{\star}+y_{1} x_{0}^{\star}+x_{0} z_{1}^{\star}+\alpha y_{1} z_{1}^{\star}\right)
$$

satisfies $\Delta_{\alpha} x=y$ and $\Delta_{\alpha}^{\star} x=z$. Hence,

$$
\left\|\Delta_{\alpha}\right\| \geq \max \left\{\frac{\|y\|}{\|x\|}, \frac{\|z\|}{\|x\|}\right\} .
$$

We show that equality holds in (2.2) for appropriate $\alpha$. Without loss of generality we may assume $\|z\| \leq\|y\|$, or, equivalently, $\left\|z_{1}\right\| \leq\left\|y_{1}\right\|$. Otherwise, we may interchange the roles of $z$ and $y$ and $\Delta$ and $\Delta^{\star}$, respectively. We consider two cases.

Case 1: $z_{1} \neq 0$. Let $\alpha=-x_{0}^{*} y /\left\|y_{1}\right\|^{2}$. Then $\Delta_{\alpha}=\widetilde{\Delta}$ and $\left\|\Delta_{\alpha}\right\|=\|y\| /\|x\|$. In order to see that let $y_{0}=y_{1} /\left\|y_{1}\right\|$ and $z_{0}=z_{1} /\left\|z_{1}\right\|$. Then

$$
\begin{aligned}
\Delta_{\alpha} & =\|x\|^{-1}\left[\begin{array}{ll}
x_{0} & y_{0}
\end{array}\right]\left[\begin{array}{cc}
x_{0}^{*} y & \left\|z_{1}\right\| \\
\left\|y_{1}\right\| & \alpha\left\|y_{1}\right\|\left\|z_{1}\right\|
\end{array}\right]\left[\begin{array}{ll}
x_{0} & z_{0}
\end{array}\right]^{\star} \\
& =\frac{\|y\|}{\|x\|}\left[\begin{array}{ll}
x_{0} & y_{0}
\end{array}\right] \underbrace{\frac{1}{\|y\|}\left[\begin{array}{cc}
x_{0}^{*} y & \left\|y_{1}\right\| \\
\left\|y_{1}\right\| & -x_{0}^{*} y
\end{array}\right]}_{=: C} \underbrace{\left[\begin{array}{cc}
1 & 0 \\
0 & \left\|z_{1}\right\| /\left\|y_{1}\right\|
\end{array}\right]}_{=: D}\left[\begin{array}{ll}
x_{0} & z_{0}
\end{array}\right]^{\star} .
\end{aligned}
$$

Since $\left[x_{0} y_{0}\right]^{*}\left[\begin{array}{ll}x_{0} & y_{0}\end{array}\right]=\left[\begin{array}{ll}x_{0} & z_{0}\end{array}\right]^{*}\left[\begin{array}{ll}x_{0} & z_{0}\end{array}\right]=I$ we have

$$
\left\|\left[\begin{array}{ll}
x_{0} & y_{0}
\end{array}\right]\right\|=\left\|\left[\begin{array}{ll}
x_{0} & z_{0}
\end{array}\right]^{\star}\right\|=1
$$

The matrix $C$ is easily seen to be unitary. Moreover, $\|D\|=\max \left\{1,\left\|z_{1}\right\| /\left\|y_{1}\right\|\right\}=1$. Consequently, $\left\|\Delta_{\alpha}\right\| \leq\|y\| /\|x\|$. This inequality is actually an equality because of (2.2).

Case $2: z_{1}=0$. Then for any $\alpha, \Delta_{\alpha}=\|x\|^{-1} y x_{0}^{*}$, whence $\left\|\Delta_{\alpha}\right\|=\|y\| /\|x\|$.

3. Reformulation of the problem. We first reformulate the original Problem 2.3 of finding the backward error for the $\star$-palindromic polynomial into an equivalent problem of maximizing the Rayleigh quotient of a Hermitian matrix with respect to some constraints. These constraints involve Hermitian matrices when $\star=*$ and symmetric matrices when $\star=T$. Let $\lambda \in \mathbb{C} \backslash\{0\}$, let $P(z)=\sum_{j=0}^{m} z^{j} A_{j}$ be a $\star$-palindromic matrix polynomial such that $M=P(\lambda)^{-1}$ exists and define $v_{\lambda}=\sum_{j=0}^{m} \lambda^{j} v_{j}$, where $v_{0}, \ldots, v_{m} \in \mathbb{C}^{n}$. Also let $k=\left\lfloor\frac{m-1}{2}\right\rfloor$.

Copyright $@$ by SIAM. Unauthorized reproduction of this article is prohibited. 
By Theorem 2.5 for any $v_{0}, \ldots, v_{m} \in \mathbb{C}^{n}$ that satisfy $v_{\lambda} \neq 0$, there exists a $\Delta=\left(\Delta_{0}, \ldots, \Delta_{m}\right) \in$ pal $_{\star}$ such that

$$
\Delta_{j} M v_{\lambda}=v_{j} \text { and } \Delta_{j}^{\star} M v_{\lambda}=v_{m-j}, j=0, \ldots, k,
$$

if and only if $\left(M v_{\lambda}\right)^{\star} v_{j}=v_{m-j}^{\star}\left(M v_{\lambda}\right)$. For any $\Delta_{j} \in \mathbb{C}^{n \times n}$ satisfying (3.1) which is minimal with respect to the 2-norm, we have $\left\|\Delta_{j}\right\|=\max \left\{\frac{\left\|v_{j}\right\|}{\left\|M v_{\lambda}\right\|}, \frac{\left\|v_{m-j}\right\|}{\left\|M v_{\lambda}\right\|}\right\}$.

If $m$ is even, the matrix $\Delta_{\frac{m}{2}}$ of the tuple $\boldsymbol{\Delta}=\left(\Delta_{0}, \ldots, \Delta_{m}\right)$ is Hermitian when $\star=*$ and symmetric when $\star=T$. In the case $\star=*$, the Hermitian matrix $\Delta_{\frac{m}{2}}$ may be chosen to satisfy $\Delta_{\frac{m}{2}} M v_{\lambda}=v_{\frac{m}{2}}$ if and only if $\left(M v_{\lambda}\right)^{*} v_{\frac{m}{2}} \in \mathbb{R}$ (for details, see [17]). On the other hand, when $\star=T$ the symmetric matrix $\Delta_{\frac{m}{2}}$ may be chosen to satisfy $\Delta_{\frac{m}{2}} M v_{\lambda}=v_{\frac{m}{2}}$ without any restrictions on $M v_{\lambda}$ and $v_{\frac{m}{2}}$, and in either case, any minimal 2-norm solution of this mapping problem satisfies $\left\|\Delta_{\frac{m}{2}}\right\|=\frac{\| v_{m} / 2}{\left\|M v_{\lambda}\right\|}$ (see [24]). Therefore, if all the constraints are fulfilled, the minimal norm of $\Delta$ is given by

$$
\|\boldsymbol{\Delta}\|_{w}^{2}=f\left(v_{0}, \ldots, v_{m}\right),
$$

where

$$
f\left(v_{0}, \ldots, v_{m}\right):= \begin{cases}\sum_{j=0}^{k} 2 w_{j}^{2} \max \left\{\frac{\left\|v_{j}\right\|^{2}}{\left\|M v_{\lambda}\right\|^{2}}, \frac{\left\|v_{m-j}\right\|^{2}}{\left\|M v_{\lambda}\right\|^{2}}\right\} & \text { if } m \text { is odd } \\ \sum_{j=0}^{k} 2 w_{j}^{2} \max \left\{\frac{\left\|v_{j}\right\|^{2}}{\left\|M v_{\lambda}\right\|^{2}}, \frac{\left\|v_{m-j}\right\|^{2}}{\left\|M v_{\lambda}\right\|^{2}}\right\}+w_{\frac{m}{2}}^{2} \frac{\left\|v_{\frac{m}{2}}\right\|^{2}}{\left\|M v_{\lambda}\right\|^{2}} & \text { if } m \text { is even. }\end{cases}
$$

Thus, Lemma 2.4 yields

$$
\eta_{w}^{\mathrm{pal}_{\star}}(P, \lambda)^{2}=\inf \left\{f\left(v_{0}, \ldots, v_{m}\right) \mid\left(v_{0}, \ldots, v_{m}\right) \in \mathcal{K}\right\},
$$

where $\mathcal{K} \subseteq\left(\mathbb{C}^{n}\right)^{m+1}$ is given by

$$
\mathcal{K}:=\left\{\left(v_{0}, \ldots, v_{m}\right) \mid v_{\lambda} \neq 0,\left(M v_{\lambda}\right)^{\star} v_{j}=v_{m-j}^{\star} M v_{\lambda}, j=0 \ldots, k\right\}
$$

if $\star=T$ or if $m$ is odd and $\star=*$ and by

$$
\mathcal{K}:=\left\{\left(v_{0}, \ldots, v_{m}\right) \mid v_{\lambda} \neq 0,\left(M v_{\lambda}\right)^{*} v_{\frac{m}{2}} \in \mathbb{R},\left(M v_{\lambda}\right)^{*} v_{j}=v_{m-j}^{*} M v_{\lambda}, j=0, \ldots, k\right\}
$$

otherwise (i.e., when $\star=*$ and $m$ is even). Observe that $\left(M v_{\lambda}\right)^{\star} v_{j}=v_{m-j}^{\star}\left(M v_{\lambda}\right)$ for $j=0, \ldots, k$ if and only if

$$
0=\left(M\left(v_{0}+\cdots+\lambda^{m} v_{m}\right)\right)^{\star} v_{j}-v_{m-j}^{\star}\left(M\left(v_{0}+\cdots+\lambda^{m} v_{m}\right)\right)=v^{\star} \widetilde{C}_{j} v,
$$

where $v:=\left[v_{0}^{T}, \ldots, v_{m}^{T}\right]^{T}$ and

$$
\widetilde{C}_{j}:=\left(\Lambda_{m}^{\star} e_{j+1}^{\star}\right) \otimes M^{\star}-\left(e_{m-j+1} \Lambda_{m}\right) \otimes M,
$$

with $\Lambda_{m}:=\left[1, \lambda, \ldots, \lambda^{m}\right] \in \mathbb{C}^{1 \times(m+1)}$. Similarly $\left(M v_{\lambda}\right)^{*} v_{\frac{m}{2}} \in \mathbb{R}$ if and only if

$$
0=-2 \operatorname{Im}\left(\left(M v_{\lambda}\right)^{*} v \frac{m}{2}\right)=i\left(v_{\frac{m}{2}}^{*}\left(M v_{\lambda}\right)-\left(M v_{\lambda}\right)^{*} v \frac{m}{2}\right)=v^{*} \widetilde{C}_{\frac{m}{2}} v
$$

Copyright (c) by SIAM. Unauthorized reproduction of this article is prohibited. 
where

$$
\widetilde{C}_{\frac{m}{2}}:=i\left(\left(\Lambda_{m}^{*} e_{\frac{m}{2}+1}^{*}\right) \otimes M^{*}-\left(e_{\frac{m}{2}+1} \Lambda_{m}\right) \otimes M\right)
$$

Note that $\widetilde{C}_{\frac{m}{2}}$ is a Hermitian matrix but the matrices $\widetilde{C}_{j}, j=0, \ldots, k$, are not Hermitian. Thus, from (3.3) if $\star=T$, or if $m$ is odd and $\star=*$,

$$
\mathcal{K}=\left\{\left(v_{0}, \ldots, v_{m}\right) \mid v_{\lambda} \neq 0, v^{\star} \widetilde{C}_{j} v=0, j=0, \ldots, k\right\},
$$

and from (3.4)

$$
\mathcal{K}=\left\{\left(v_{0}, \ldots, v_{m}\right) \mid v_{\lambda} \neq 0, v^{*} \widetilde{C}_{\frac{m}{2}} v=0, v^{*} \widetilde{C}_{j} v=0, j=0, \ldots, k\right\}
$$

otherwise.

As stated in the beginning of this section, our aim is to reformulate the computation of the structured eigenvalue backward error as an equivalent problem of maximizing the Rayleigh quotient of a Hermitian matrix subject to some constraints. The same strategy was applied in [6] to find the structured eigenvalue backward error $\eta_{w}^{\mathbb{S}}(P, \lambda)$ for Hermitian and related structures. But the reformulation was aided by the fact that $\eta_{w}^{\mathbb{S}}(P, \lambda)$ satisfied

$$
\eta_{w}^{\mathbb{S}}(P, \lambda)=\left(\sup \left\{\frac{\left\|M v_{\lambda}\right\|^{2}}{\sum_{j=0}^{m} w_{j}^{2}\left\|v_{j}\right\|^{2}} \mid v_{\lambda} \neq 0, v_{j}^{*} M v_{\lambda} \in \mathbb{R}\right\}\right)^{-\frac{1}{2}}
$$

for those structures, because the quotient in the right-hand side of (3.9) could easily be seen to be a Rayleigh quotient of a particular Hermitian matrix. (For details, we refer to the proof of [6, Theorem 4.4].) However, as (3.2) suggests, this is not the case for the structured eigenvalue backward error $\eta_{w}^{\mathrm{pal}_{\star}}(P, \lambda)$ for the $\star$-palindromic structures, because the function $f$ in the right-hand side of (3.2) involves taking a maximum. The following lemma is a key step toward establishing a relationship similar to (3.9) for $\eta_{w}^{\text {pal }_{\star}}(P, \lambda)$, because it shows that minimizers of the function $f$ in (3.2) also minimize a related function $g$ that can in the following be interpreted as a Rayleigh quotient of a certain Hermitian matrix.

Lemma 3.1. Let $P(z)=\sum_{j=0}^{m} z^{j} A_{j}$ be $\star$-palindromic and $\lambda \in \mathbb{C} \backslash\{0\}$. Assume further that $M=(P(\lambda))^{-1}$ exists and $k=\left\lfloor\frac{m-1}{2}\right\rfloor$. Then

$$
\eta_{w}^{\mathrm{pal}_{\star}}(P, \lambda)^{2}=\inf \left\{g\left(v_{0}, \ldots, v_{m}\right) \mid\left(v_{0}, \ldots, v_{m}\right) \in \mathcal{K}\right\},
$$

where

$$
g\left(v_{0}, \ldots, v_{m}\right):= \begin{cases}\sum_{j=0}^{k} \frac{2 w_{j}^{2}\left(\left\|v_{j}\right\|^{2}+|\lambda|^{m-2 j}\left\|v_{m-j}\right\|^{2}\right)}{\left(1+|\lambda|^{m-2 j}\right)\left\|M v_{\lambda}\right\|^{2}} & \text { if } m \text { is odd }, \\ \sum_{j=0}^{k} \frac{2 w_{j}^{2}\left(\left\|v_{j}\right\|^{2}+|\lambda|^{m-2 j}\left\|v_{m-j}\right\|^{2}\right)}{\left(1+|\lambda|^{m-2 j}\right)\left\|M v_{\lambda}\right\|^{2}}+\frac{w_{\frac{m}{2}}^{2}\left\|v v_{\frac{m}{2}}\right\|^{2}}{\left\|M v_{\lambda}\right\|^{2}} & \text { if } m \text { is even },\end{cases}
$$

and $\mathcal{K}$ is as defined in (3.7) and (3.8), respectively. 
Proof. Set $\nu:=\inf \left\{g\left(v_{0}, \ldots, v_{m}\right) \mid\left(v_{0}, \ldots, v_{m}\right) \in \mathcal{K}\right\}$. It is easily verified that $g\left(v_{0}, \ldots, v_{m}\right) \leq f\left(v_{0}, \ldots, v_{m}\right)$ for all $\left(v_{0}, \ldots, v_{m}\right) \in\left(\mathbb{C}^{n}\right)^{m+1}$ with $v_{\lambda} \neq 0$. This together with (3.2) implies $\nu \leq \eta_{w}^{\mathrm{pal}_{\star}}(P, \lambda)^{2}$. The opposite inequality is an immediate consequence of the following facts:

(a) The infimum of $g$ in the definition of $\nu$ is attained for some $\left(\hat{v}_{0}, \ldots, \hat{v}_{m}\right) \in \mathcal{K}$.

(b) For every minimizer $\left(\hat{v}_{0}, \ldots, \hat{v}_{m}\right) \in \mathcal{K}$ of $g$ we have

$$
g\left(\hat{v}_{0}, \ldots, \hat{v}_{m}\right)=f\left(\hat{v}_{0}, \ldots, \hat{v}_{m}\right)
$$

Proof of (a). Because $\mathcal{K}$ is closed under scalar multiplication and since for all $t \in \mathbb{R} \backslash\{0\}$ and all $\left(v_{0}, \ldots, v_{m}\right) \in \mathcal{K}$ we have $g\left(v_{0}, \ldots, v_{m}\right)=g\left(t v_{0}, \ldots, t v_{m}\right)$, we obtain that $g(\mathcal{K})=g(\mathcal{K} \cap \mathcal{S})$, where $\mathcal{S}$ is defined as

$$
\mathcal{S}=\left\{\left(v_{0}, \ldots, v_{m}\right) \in\left(\mathbb{C}^{n}\right)^{m+1} \mid \sum_{j=0}^{k}\left\|v_{j}\right\|^{2}=1\right\}
$$

Let $\left(v_{0}^{(\ell)}, \ldots, v_{m}^{(\ell)}\right), \ell \in \mathbb{N}$, be a sequence in $\mathcal{K} \cap \mathcal{S}$ for which

$$
\lim _{\ell \rightarrow \infty} g\left(v_{0}^{(\ell)}, \ldots, v_{m}^{(\ell)}\right)=\nu
$$

Since $\mathcal{S}$ is compact we may assume without loss of generality that the sequence $\left(v_{0}^{(\ell)}, \ldots, v_{m}^{(\ell)}\right)$ has a limit $\left(\hat{v}_{0}, \ldots, \hat{v}_{m}\right) \in \mathcal{S}$. Suppose that $\left(\hat{v}_{0}, \ldots, \hat{v}_{m}\right) \notin \mathcal{K}$. Then we have $\hat{v}_{0}+\lambda v_{1}+\cdots+\lambda^{m} \hat{v}_{m}=0$, as $\left(\hat{v}_{0}, \ldots, \hat{v}_{m}\right)$ belongs to the closure of $\mathcal{K}$. This implies

$$
\lim _{\ell \rightarrow \infty}\left\|M\left(v_{0}^{(\ell)}+\lambda v_{1}^{(\ell)}+\cdots+\lambda^{m} v_{m}^{(\ell)}\right)\right\|^{-1}=\infty
$$

and hence

$$
\lim _{\ell \rightarrow \infty} g\left(v_{0}^{(\ell)}, \ldots, v_{m}^{(\ell)}\right)=\infty \neq \nu
$$

which is a contradiction. Thus, $\left(\hat{v}_{0}, \ldots, \hat{v}_{m}\right) \in \mathcal{K}$ and $g\left(\hat{v}_{0}, \ldots, \hat{v}_{m}\right)=\nu$.

Proof of $(\mathrm{b})$. Let $\left(\hat{v}_{0}, \ldots, \hat{v}_{m}\right) \in \mathcal{K}$ be such that $g\left(\hat{v}_{0}, \ldots, \hat{v}_{m}\right)=\nu$. Observe that, to show that $g\left(\hat{v}_{0}, \ldots, \hat{v}_{m}\right)=f\left(\hat{v}_{0}, \ldots, \hat{v}_{m}\right)$, it is sufficient to show that $\left\|\hat{v}_{j}\right\|=\left\|\hat{v}_{m-j}\right\|$ for all $j=0, \ldots, k$. Let

$$
x_{0}= \begin{cases}M\left(\hat{v}_{\lambda}\right) /\left\|M\left(\hat{v}_{\lambda}\right)\right\| & \text { if } \star=*, \\ \hline M\left(\hat{v}_{\lambda}\right) /\left\|M\left(\hat{v}_{\lambda}\right)\right\| & \text { if } \star=T,\end{cases}
$$

and $y_{j}, y_{m-j}$ be the projections of $\hat{v}_{j}$ and $\hat{v}_{m-j}$, respectively, onto the orthogonal complement of $x_{0}$, for $0 \leq j \leq k$. Then

$$
\hat{v}_{j}=y_{j}+c_{j} x_{0} \quad \text { and } \quad \hat{v}_{m-j}=y_{m-j}+c_{m-j} x_{0}
$$

for some $c_{j}, c_{m-j} \in \mathbb{C}$. Since $\left(\hat{v}_{0}, \ldots, \hat{v}_{m}\right) \in \mathcal{K}$ we have $\bar{c}_{j}=c_{m-j}$ when $\star=*$ and $c_{j}=c_{m-j}$ when $\star=T$. Hence

$$
\left\|\hat{v}_{j}\right\|^{2}=\left\|y_{j}\right\|^{2}+\left|c_{j}\right|^{2} \text { and }\left\|\hat{v}_{m-j}\right\|^{2}=\left\|y_{m-j}\right\|^{2}+\left|c_{j}\right|^{2} .
$$

Copyright ( by SIAM. Unauthorized reproduction of this article is prohibited. 
Let $y=\bar{\lambda}^{m-2 j} y_{j}+|\lambda|^{m-2 j} y_{m-j}$. Observe that

$$
\left(\hat{v}_{0}, \ldots, \hat{v}_{j}+t \lambda^{m-2 j} y, \ldots, \hat{v}_{m-j}-t y, \ldots, \hat{v}_{m}\right) \in \mathcal{K}
$$

for all $t \in \mathbb{R}$. Thus as $\left(\hat{v}_{0}, \ldots, \hat{v}_{m}\right)$ is a minimizer of $g$ over $\mathcal{K}$, we have

$$
\begin{aligned}
0 & =\left.\frac{d}{d t} g\left(\hat{v}_{0}, \ldots, \hat{v}_{j}+t \lambda^{m-2 j} y, \ldots, \hat{v}_{m-j}-t y, \ldots, \hat{v}_{m}\right)\right|_{t=0} \\
& =\left.\frac{d}{d t}\left(\frac{2 w_{j}^{2}\left(\left\|\hat{v}_{j}+t \lambda^{m-2 j} y\right\|^{2}+|\lambda|^{m-2 j}\left\|\hat{v}_{m-j}-t y\right\|^{2}\right)}{\left(1+|\lambda|^{m-2 j}\right)\left\|M \hat{v}_{\lambda}\right\|^{2}}\right)\right|_{t=0} \\
& =\frac{2 w_{j}^{2} \Re\left(\hat{v}_{j}^{*}\left(\lambda^{m-2 j} y\right)-|\lambda|^{m-2 j} \hat{v}_{m-j}^{*} y\right)}{\left(1+|\lambda|^{m-2 j}\right)\left\|M \hat{v}_{\lambda}\right\|^{2}} \quad\left(\text { since }\left.\frac{d}{d t}\|v+t y\|^{2}\right|_{t=0}=2 \Re\left(v^{*} y\right)\right) \\
& =\frac{2 w_{j}^{2} \Re\left(y_{j}^{*}\left(\lambda^{m-2 j} y\right)-|\lambda|^{m-2 j} y_{m-j}^{*} y\right)}{\left(1+|\lambda|^{m-2 j}\right)\left\|M \hat{v}_{\lambda}\right\|^{2}} \\
& =\frac{2 w_{j}^{2}|\lambda|^{m-2 j} \Re\left(|\lambda|^{m-2 j}\left\|y_{j}\right\|^{2}+\lambda^{m-2 j} y_{j}^{*} y_{m-j}-\bar{\lambda}^{m-2 j} y_{m-j}^{*} y_{j}-|\lambda|^{m-2 j}\left\|y_{m-j}\right\|^{2}\right)}{\left(1+|\lambda|^{m-2 j}\right)\left\|M \hat{v}_{\lambda}\right\|^{2}} \\
& =\frac{2 w_{j}^{2}|\lambda|^{2(m-2 j)}\left(\left\|y_{j}\right\|^{2}-\left\|y_{m-j}\right\|^{2}\right)}{\left(1+|\lambda|^{m-2 j}\right)\left\|M \hat{v}_{\lambda}\right\|^{2}},
\end{aligned}
$$

which implies $\left\|y_{j}\right\|=\left\|y_{m-j}\right\|$. This together with (3.10) yields $\left\|\hat{v}_{j}\right\|=\left\|\hat{v}_{m-j}\right\|$. Hence $\left\|\hat{v}_{j}\right\|=\left\|\hat{v}_{m-j}\right\|$ for all $j$, and the latter implies $g\left(\hat{v}_{0}, \ldots, \hat{v}_{m}\right)=f\left(\hat{v}_{0}, \ldots, \hat{v}_{m}\right)$. This completes the proof.

Recalling that $k=\left\lfloor\frac{m-1}{2}\right\rfloor$, define $\gamma_{j 1}=w_{j} \sqrt{\frac{2}{1+|\lambda|^{m-2 j}}}, \gamma_{j 2}=w_{j} \sqrt{\frac{2|\lambda|^{m-2 j}}{1+|\lambda|^{m-2 j}}}$, $j=0, \ldots, k$, and

$$
\Gamma:= \begin{cases}\operatorname{diag}\left(\gamma_{01}, \ldots, \gamma_{k 1}, \gamma_{k 2}, \ldots, \gamma_{02}\right) \otimes I_{n} & \text { if } m \text { is odd } \\ \operatorname{diag}\left(\gamma_{01}, \ldots, \gamma_{k 1}, w_{\frac{m}{2}}, \gamma_{k 2}, \ldots, \gamma_{02}\right) \otimes I_{n} & \text { if } m \text { is even. }\end{cases}
$$

Also recall that $\Lambda_{m}=\left[1, \lambda, \ldots, \lambda^{m}\right] \in \mathbb{C}^{1 \times(m+1)}$. Then we have

$$
g\left(v_{0}, \ldots, v_{m}\right)=\frac{v^{*} \Gamma^{2} v}{v^{*} \widetilde{G} v}, \quad \text { where } \widetilde{G}:=\left(\Lambda_{m}^{*} \Lambda_{m}\right) \otimes\left(M^{*} M\right), \quad v=\left[v_{0}^{T}, \ldots, v_{m}^{T}\right]^{T},
$$

and $v^{*} \widetilde{G} v=\left\|M v_{\lambda}\right\|^{2} \neq 0$, or, equivalently, $v_{\lambda} \neq 0$. It follows that

$$
\begin{aligned}
\eta_{w}^{\mathrm{pal}_{\star}}(P, \lambda) & =\left(\inf \left\{f\left(v_{0}, \ldots, v_{m}\right) \mid\left(v_{0}, \ldots, v_{m}\right) \in \mathcal{K}\right\}\right)^{1 / 2} \\
& =\left(\inf \left\{g\left(v_{0}, \ldots, v_{m}\right) \mid\left(v_{0}, \ldots, v_{m}\right) \in \mathcal{K}\right\}\right)^{1 / 2} \\
& =\left(\sup \left\{g\left(v_{0}, \ldots, v_{m}\right)^{-1} \mid\left(v_{0}, \ldots, v_{m}\right) \in \mathcal{K}\right\}\right)^{-1 / 2} .
\end{aligned}
$$

Set $u:=\Gamma v$ and

$$
G:=\Gamma^{-1} \widetilde{G} \Gamma^{-1}, \quad C_{j}:=\Gamma^{-1} \widetilde{C}_{j} \Gamma^{-1}, \quad C_{\frac{m}{2}}:=\Gamma^{-1} \widetilde{C}_{\frac{m}{2}} \Gamma^{-1},
$$

where $\widetilde{G}, \widetilde{C}_{j}$, and $\widetilde{C}_{\frac{m}{2}}$ are as defined in (3.11), (3.5), and (3.6), respectively.

Copyright $@$ by SIAM. Unauthorized reproduction of this article is prohibited. 
By Lemma 3.1 and (3.11), for $k=\left\lfloor\frac{m-1}{2}\right\rfloor$, we have

$$
\begin{aligned}
\left(\eta_{w}^{\mathrm{pal}_{\star}}(P, \lambda)\right)^{-2} & =\sup \left\{\frac{v^{*} \widetilde{G} v}{v^{*} \Gamma^{2} v} \mid v \in \mathbb{C}^{n(m+1)} \backslash\{0\}, v^{\star} \widetilde{C}_{j} v=0, j=0, \ldots, k\right\} \\
& =\sup \left\{\frac{u^{*} G u}{u^{*} u} \mid u \in \mathbb{C}^{n(m+1)} \backslash\{0\}, u^{\star} C_{j} u=0, j=0, \ldots, k\right\}
\end{aligned}
$$

if $\star=T$, or if $m$ is odd and $\star=*$, and

$$
\left(\eta_{w}^{\mathrm{pal}_{*}}(P, \lambda)\right)^{-2}=\sup \left\{\frac{u^{*} G u}{u^{*} u} \mid u \in \mathbb{C}^{n(m+1)} \backslash\{0\}, u^{*} C_{\frac{m}{2}} u=0, u^{*} C_{j} u=0, j=0, \ldots, k\right\}
$$

otherwise. Note that the condition $v_{\lambda} \neq 0$ from the definition of $\mathcal{K}$ in (3.7) or (3.8), respectively, or, equivalently, the conditions $v^{*} \widetilde{G} v \neq 0$ and $u^{*} G u \neq 0$ can be dropped in the two expressions for $\left(\eta_{w}^{\mathrm{pal}_{*}}(P, \lambda)\right)^{-2}$, because $\widetilde{G}$ and $G$ are semidefinite. This implies $\frac{u^{*} G u}{u^{*} u} \geq 0$ and hence the supremum of this Rayleigh quotient over all nonzero vectors $u$ satisfying some constraints will be the same with or without the additional condition $u^{*} G u \neq 0$.

In order to state the main result of this section, for each $j=0, \ldots, k$ we define

$$
\begin{aligned}
H_{j}:=C_{j}+C_{j}^{*}, H_{m-j} & :=i\left(C_{j}-C_{j}^{*}\right), H_{\frac{m}{2}}:=C_{\frac{m}{2}}, \\
S_{j} & :=C_{j}+C_{j}^{T},
\end{aligned}
$$

where $C_{j}$, for $j=0, \ldots, k$, and $C_{\frac{m}{2}}$, are as in (3.12).

Observe that for $j=0, \ldots, k$,

$$
\begin{aligned}
v^{*} \widetilde{C}_{j} v & =0 \Longleftrightarrow u^{*} H_{j} u=0 \text { and } u^{*} H_{m-j} u=0, \\
v^{T} \widetilde{C}_{j} v & =0 \Longleftrightarrow u^{T} S_{j} u=0, \\
v^{*} \widetilde{C}_{\frac{m}{2}} v & =0 \Longleftrightarrow u^{*} H_{\frac{m}{2}} u=0 .
\end{aligned}
$$

Therefore we have proved the following theorem, which gives the desired reformulation.

Theorem 3.2. Let $P(z)=\sum_{j=0}^{m} z^{j} A_{j}$ be $\star$-palindromic and $\lambda \in \mathbb{C} \backslash\{0\}$. Suppose that $P(\lambda)$ is nonsingular and $M=(P(\lambda))^{-1}$. Furthermore, let $k:=\left\lfloor\frac{m-1}{2}\right\rfloor, G$ be as in (3.12), $H_{j}$, for $j=0, \ldots, m$, be defined by (3.13), and $S_{j}$, for $j=0, \ldots, k$, be defined by (3.14). Then

$$
\eta_{w}^{\mathrm{pal}_{\mathrm{T}}}(P, \lambda)=\left(\sup \left\{\frac{u^{*} G u}{u^{*} u} \mid u \in \mathbb{C}^{n(m+1)} \backslash\{0\}, u^{T} S_{j} u=0, j=0, \ldots, k\right\}\right)^{-\frac{1}{2}}
$$

and

$$
\eta_{w}^{\mathrm{pal}_{*}}(P, \lambda)=\left(\sup \left\{\frac{u^{*} G u}{u^{*} u} \mid u \in \mathbb{C}^{n(m+1)} \backslash\{0\}, u^{*} H_{j} u=0, j=0, \ldots, m\right\}\right)^{-\frac{1}{2}}
$$

4. Backward errors for approximate eigenvalues of *-palindromic matrix polynomials. In this section, we obtain structured eigenvalue backward errors $\eta_{w}^{\text {pal }_{*}}(\lambda, P)$ for matrix polynomials $P(z)$ with $*$-palindromic structure. As mentioned in section 1 , if $\lambda \in \mathbb{C} \backslash\{0\}$ is such that $|\lambda|=1$, then there is no difference between the eigenvalue backward errors with respect to structure preserving and arbitrary

Copyright $@$ by SIAM. Unauthorized reproduction of this article is prohibited. 
perturbations. This fact was shown in [1] for the weight vector $w=(1, \ldots, 1)$ and easily generalizes to arbitrary choices of palindromic weight vectors. The situation is completely different if $|\lambda| \neq 1$. In this case, we obtain the structured backward error via minimization of the maximal eigenvalue of a parameter-dependent Hermitian matrix. The following theorem, which is a combination of Theorem 3.2 and Theorem 3.5 of [6], is crucial to this process. Note that in this theorem "indefinite" means "strictly not semidefinite" as opposed to "not necessarily definite" as used in [7].

Theorem 4.1. Let $G, H_{0}, \ldots, H_{p} \in \mathbb{C}^{n \times n}$ be Hermitian. Assume that any nonzero linear combination $\alpha_{0} H_{0}+\cdots+\alpha_{p} H_{p},\left(\alpha_{0}, \ldots, \alpha_{p}\right) \in \mathbb{R}^{p+1} \backslash\{0\}$, is indefinite. Then the following statements hold:

(1) The function $L: \mathbb{R}^{p+1} \rightarrow \mathbb{R},\left(t_{0}, \ldots, t_{p}\right) \mapsto \lambda_{\max }\left(G+t_{0} H_{0}+\cdots+t_{p} H_{p}\right)$ is convex and has a global minimum

$$
\lambda_{\max }^{\bullet}=\min _{t_{0}, \ldots, t_{p} \in \mathbb{R}} L\left(t_{0}, \ldots, t_{p}\right) .
$$

(2) If either $p=1$, or the minimum $\lambda_{\max }^{\bullet}$ of $L$ is attained at $\left(\hat{t}_{0}, \ldots, \hat{t}_{p}\right) \in \mathbb{R}^{p+1}$ and is a simple eigenvalue of $H_{\bullet}:=G+\hat{t}_{0} H_{0}+\cdots+\hat{t}_{p} H_{p}$, then there exists an eigenvector $u \in \mathbb{C}^{n} \backslash\{0\}$ of $H_{\bullet}$ associated with $\lambda_{\max }^{\bullet}$ satisfying

$$
u^{*} H_{j} u=0 \quad \text { for } j=0, \ldots, p .
$$

(3) Under the assumptions of (2) we have

$$
\sup \left\{\frac{u^{*} G u}{u^{*} u} \mid u \neq 0, u^{*} H_{j} u=0, j=0, \ldots, p\right\}=\lambda_{\max }^{\bullet} .
$$

In particular, the supremum of the left-hand side of (4.2) is a maximum and is attained for the eigenvector $u$ from (4.1).

As seen in [6, Theorem 3.5], the assumption that $\lambda_{\max }^{\bullet}$ is simple is not necessary when $p=1$. This is due to the fact that the joint numerical range

$$
\mathcal{W}_{0}\left(F_{0}, F_{1}\right):=\left\{\left(x^{*} F_{0} x, x^{*} F_{1} x\right) \in \mathbb{R}^{2} \mid x \in \mathbb{C}^{\ell},\|x\|=1\right\}
$$

of two Hermitian matrices $F_{0}, F_{1} \in \mathbb{C}^{\ell \times \ell}$ is a convex set [11]. But as [6, Example 3.8] shows, the joint numerical range of three or more complex Hermitian matrices need not be convex. Hence the assumption of simplicity of $\lambda_{\max }^{\bullet}$ is essential when $p>1$. We have the following result, which gives a formula for $\eta_{w}^{\text {pal }}{ }_{*}(P, \lambda)$ when $|\lambda| \neq 1$.

Theorem 4.2. Let $P(z)=\sum_{j=0}^{m} z^{j} A_{j}$ be $*$-palindromic and $\lambda \in \mathbb{C} \backslash\{0\}$ such that $|\lambda| \neq 1$. Suppose that $P(\lambda)$ is nonsingular and $M=(P(\lambda))^{-1}$. Then for $G$ as defined in (3.12) and $H_{j}$, for $j=0, \ldots, m$, as defined in (3.13), we have that

$$
\lambda_{\max }^{\bullet}:=\min _{t_{0}, \ldots, t_{m} \in \mathbb{R}} \lambda_{\max }\left(G+t_{0} H_{0}+\cdots+t_{m} H_{m}\right)
$$

is attained for some $\left(\hat{t}_{0}, \ldots, \hat{t}_{m}\right) \in \mathbb{R}^{m+1}$. If $m=1$ or $\lambda_{\max }^{\bullet}$ is a simple eigenvalue of $G+t_{0}^{*} H_{0}+\cdots+t_{m}^{*} H_{m}$, then

$$
\eta_{w}^{\mathrm{pal}_{*}}(P, \lambda)=\frac{1}{\sqrt{\lambda_{\max }^{\bullet}}}=\left(\min _{t_{0}, \ldots, t_{m} \in \mathbb{R}} \lambda_{\max }\left(G+t_{0} H_{0}+\cdots+t_{m} H_{m}\right)\right)^{-1 / 2} .
$$

Copyright (c) by SIAM. Unauthorized reproduction of this article is prohibited. 
Proof. Setting $k=\left\lfloor\frac{m-1}{2}\right\rfloor$ let $\widetilde{H}_{j}=\widetilde{C}_{j}+\widetilde{C}_{j}^{*}, \widetilde{H}_{m-j}=i\left(\widetilde{C}_{j}-\widetilde{C}_{j}^{*}\right)$, and $\widetilde{H}_{\frac{m}{2}}=\widetilde{C}_{\frac{m}{2}}$, where $\widetilde{C}_{j}$ for $j=0, \ldots, k$ are as defined in (3.5) and (3.6). In view of Theorem 3.2, we aim to apply Theorem 4.1 in the proof. Thus we check whether each nontrivial linear combination of $H_{0}, \ldots, H_{m}$ or, equivalently, of $\widetilde{H}_{0}, \ldots, \widetilde{H}_{m}$ is indefinite. Let $H:=\sum_{j=0}^{m} \alpha_{j} \widetilde{H}_{j}$. Recalling that $\Lambda_{m}:=\left[1, \lambda, \ldots, \lambda^{m}\right] \in \mathbb{C}^{1 \times(m+1)}$, easy calculations show that

$$
H=\left(\Lambda_{m}^{*} \alpha^{*}\right) \otimes M^{*}+\left(\alpha \Lambda_{m}\right) \otimes M,
$$

where $\alpha:=\left[\alpha_{0}-i \alpha_{m}, \ldots, \alpha_{k}-i \alpha_{m-k},-\left(\alpha_{k}+i \alpha_{m-k}\right), \ldots,-\left(\alpha_{0}+i \alpha_{m}\right)\right]^{T}$ if $m$ is odd and

$$
\alpha=\left[\alpha_{0}-i \alpha_{m}, \ldots, \alpha_{k}-i \alpha_{m-k},-i \alpha_{\frac{m}{2}},-\left(\alpha_{k}+i \alpha_{m-k}\right), \ldots,-\left(\alpha_{0}+i \alpha_{m}\right)\right]^{T}
$$

if $m$ is even. To complete the proof, we show that if $H$ is semidefinite, then $\alpha=0$ and hence $\alpha_{0}=\cdots=\alpha_{m}=0$. Let

$$
Q:=\left[\begin{array}{ccccc}
1 & -\lambda & 0 & \cdots & 0 \\
0 & 1 & -\lambda & \ddots & \vdots \\
\vdots & \ddots & \ddots & \ddots & 0 \\
\vdots & & \ddots & \ddots & -\lambda \\
0 & \cdots & \cdots & 0 & 1
\end{array}\right] \in \mathbb{C}^{(m+1) \times(m+1)}
$$

and $a=\left[a_{0} \ldots, a_{m}\right]^{T}:=Q^{*} \alpha$. Since $\Lambda_{m} Q=e_{1}^{*}$ we have

$$
\begin{aligned}
\left(Q \otimes I_{n}\right)^{*} H\left(Q \otimes I_{n}\right) & =\left(Q^{*} \Lambda_{m}^{*} \alpha^{*} Q\right) \otimes M^{*}+\left(Q^{*} \alpha \Lambda_{m} Q\right) \otimes M \\
& =\left(e_{1} a^{*}\right) \otimes M^{*}+\left(a e_{1}^{*}\right) \otimes M \\
& =\left[\begin{array}{cccc}
a_{0} M+\bar{a}_{0} M^{*} & \bar{a}_{1} M^{*} & \ldots & \bar{a}_{m} M^{*} \\
a_{1} M & 0 & \ldots & 0 \\
\vdots & \vdots & & \vdots \\
a_{m} M & 0 & \ldots & 0
\end{array}\right] .
\end{aligned}
$$

If $H$ is semidefinite, then $a_{1}=\cdots=a_{m}=0$ and hence $Q^{*} \alpha=a=a_{0} e_{1}$. When $m \geq 3$, observing that

$$
\begin{gathered}
a_{1}=0 \Rightarrow \alpha_{1}-i \alpha_{m-1}=\bar{\lambda}\left(\alpha_{0}-i \alpha_{m}\right), \\
\bar{a}_{m}=0 \Rightarrow \alpha_{0}-i \alpha_{m}=\lambda\left(\alpha_{1}-i \alpha_{m-1}\right),
\end{gathered}
$$

we have $a_{0}=\alpha_{0}-i \alpha_{m}=\lambda\left(\alpha_{1}-i \alpha_{m-1}\right)=\lambda \bar{\lambda}\left(\alpha_{0}-i \alpha_{m}\right)=\lambda \bar{\lambda} a_{0}$.

Similarly, when $m=1$,

$$
a_{1}=0 \Rightarrow \alpha_{0}+i \alpha_{1}=-\bar{\lambda}\left(\alpha_{0}-i \alpha_{1}\right) \text { and } \alpha_{0}-i \alpha_{1}=-\lambda\left(\alpha_{0}+i \alpha_{1}\right)
$$

so that $a_{0}=\alpha_{0}-i \alpha_{1}=-\lambda\left(\alpha_{0}+i \alpha_{1}\right)=\lambda \bar{\lambda}\left(\alpha_{0}-i \alpha_{1}\right)=\lambda \bar{\lambda} a_{0}$.

Finally when $m=2$,

$$
\begin{aligned}
& a_{1}=0 \Rightarrow i \alpha_{1}=-\bar{\lambda}\left(\alpha_{0}-i \alpha_{2}\right), \\
& \bar{a}_{2}=0 \Rightarrow \alpha_{0}-i \alpha_{2}=-i \lambda \alpha_{1}
\end{aligned}
$$

so that $a_{0}=\alpha_{0}-i \alpha_{2}=-i \lambda \alpha_{1}=\lambda \bar{\lambda}\left(\alpha_{0}-i \alpha_{2}\right)=\lambda \bar{\lambda} a_{0}$. 
In all cases we have $a_{0}=\lambda \bar{\lambda} a_{0}$ and hence $a_{0}=0$ as $\lambda \bar{\lambda} \neq 1$. Therefore, $\alpha=$ $\left(Q^{*}\right)^{-1} a=0$, which implies that $\alpha_{0}=\cdots=\alpha_{m}=0$.

Remark 4.3. Although it cannot be established that $\lambda_{\max }^{\bullet}$ is always simple for the particular matrices $G, H_{0}, \ldots, H_{m}$ in Theorem 4.2, numerical experiments suggests that this holds generically.

Remark 4.4. There may be situations when it would be necessary to find the backward error $\eta_{w}^{\mathrm{pal}_{*}}(P, \lambda)$ under the restriction that $*$-palindromic perturbations can affect only some of the coefficient matrices. This is equivalent to setting some of the entries in the palindromic weight vector $w$ to zero. Let $\mathcal{I}:=\left\{j_{0}, j_{1}, \ldots, j_{\ell}\right\}$ be a subset of $\{0,1, \ldots,\lfloor m-1\rfloor / 2\}$ if $m$ is odd and of $\{0,1, \ldots, m / 2\}$ if $m$ is even and assume that $j_{0}<j_{1}<\cdots<j_{\ell}$. Suppose that $\mathcal{I}$ is the set of indices such that only the coefficients $A_{j}$ and $A_{m-j}, j \in \mathcal{I}$ of $P(z)$ are affected by perturbations. Let $\widehat{w}$ be a palindromic weight vector extracted from $w$ by retaining only its nonzero entries. Then $\widehat{w}$ belongs to $\mathbb{R}^{2 \ell+3}$ if $m$ is even and $j_{\ell}=\frac{m}{2}$ and to $\mathbb{R}^{2 \ell+2}$ otherwise. For any $\left(\Delta_{0}, \ldots, \Delta_{m}\right) \in \mathrm{pal}_{*}$ with $\Delta_{j}=\Delta_{m-j}=0$ if $j \notin \mathcal{I}$, we then have

$$
\begin{aligned}
& \left\|\left(\Delta_{0}, \ldots, \Delta_{m}\right)\right\|_{w}^{2} \\
= & \left\|\left(\Delta_{j_{0}}, \Delta_{j_{1}}, \ldots, \Delta_{m-j_{1}}, \Delta_{m-j_{0}}\right)\right\|_{\widehat{w}}^{2} \\
= & \begin{cases}\sum_{j \in \mathcal{I}}\left(w_{j}^{2}\left\|\Delta_{j}\right\|^{2}+w_{m-j}^{2}\left\|\Delta_{m-j}\right\|^{2}\right)+w_{\frac{m}{2}}^{2}\left\|\Delta_{\frac{m}{2}}\right\|^{2} & \text { if } m \text { is even and } j_{\ell}=\frac{m}{2}, \\
\sum_{j \in \mathcal{I}}\left(w_{j}^{2}\left\|\Delta_{j}\right\|^{2}+w_{m-j}^{2}\left\|\Delta_{m-j}\right\|^{2}\right) & \text { otherwise. }\end{cases}
\end{aligned}
$$

Then $\|\cdot\|_{\widehat{w}}$ defines a norm on $\left(\mathbb{C}^{n \times n}\right)^{2 \ell+3}$ in the first case and on $\left(\mathbb{C}^{n \times n}\right)^{2 \ell+2}$ in the second case. By using this, and the weight vector $\widehat{w}$, the strategy of reformulation proposed in section 3 may be used to compute the structured backward error $\eta_{w}^{\mathrm{pal}_{*}}(P, \lambda)$ with fewer constraints and smaller Hermitian matrices involved in each constraint. The details of this process are similar to those in [6, section 6] for Hermitian matrix polynomials and are therefore omitted.

Remark 4.5. To obtain an optimal *-palindromic perturbation to $P(z)$ with norm equal to the structured backward error $\eta_{w}^{\mathrm{pal}_{*}}(P, \lambda)$ such that the perturbed polynomial has an eigenvalue at $\lambda$, we first compute the eigenvector $u$ corresponding to the eigenvalue $\lambda_{\max }^{\bullet}$ of $G+\hat{t}_{0} H_{0}+\cdots+\hat{t}_{m} H_{m}$ that satisfies the constraints $u^{*} H_{j} u=0$. Setting, $v:=\Gamma^{-1} u$, the coefficient matrices $\Delta_{j}$ of the $*$-palindromic perturbation may be obtained from Theorem 2.5 and [6, Theorem 2.6], the second result being necessary only to construct $\Delta_{\frac{m}{2}}$ when $m$ is even.

To highlight the fact that the assumption of simplicity of $\lambda_{\max }^{\bullet}$ is not required when $m=1$, we state the result for the case of the $*$-palindromic pencils separately.

Theorem 4.6. Let $A \in \mathbb{C}^{n \times n}$ and $\lambda \in \mathbb{C} \backslash\{0\}$ with $|\lambda| \neq 1$. Suppose that the pencil $P(\lambda)=A+\lambda A^{*}$ is nonsingular and let $M:=\left(A+\lambda A^{*}\right)^{-1}$. Furthermore define $\gamma_{1}:=\sqrt{\frac{2}{1+|\lambda|}}, \gamma_{2}:=\sqrt{\frac{2|\lambda|}{1+|\lambda|}}$

$\widetilde{G}:=\left[\begin{array}{cc}M^{*} M & \lambda M^{*} M \\ \bar{\lambda} M^{*} M & |\lambda|^{2} M^{*} M\end{array}\right], C:=\left[\begin{array}{cc}M^{*} & 0 \\ \bar{\lambda} M^{*}-M & -\lambda M\end{array}\right], \Gamma:=\left[\begin{array}{cc}w_{0} \gamma_{1} I_{n} & 0 \\ 0 & w_{0} \gamma_{2} I_{n}\end{array}\right]$,

$$
G:=\Gamma^{-1} \widetilde{G} \Gamma^{-1}, \quad H_{0}=\Gamma^{-1}\left(C+C^{*}\right) \Gamma^{-1}, \quad \text { and } H_{1}:=i \Gamma^{-1}\left(C-C^{*}\right) \Gamma^{-1} .
$$

Then

$$
\eta_{w}^{\mathrm{pal}_{*}}(P, \lambda)=\left(\min _{t_{0}, t_{1} \in \mathbb{R}} \lambda_{\max }\left(G+t_{0} H_{0}+t_{1} H_{1}\right)\right)^{-1 / 2} .
$$

Copyright $@$ ㅇ by SIAM. Unauthorized reproduction of this article is prohibited. 
5. Structured eigenvalue backward errors for $T$-palindromic matrix polynomials. In this section, we obtain the structured eigenvalue backward error $\eta_{w}^{\mathrm{pal}_{\mathrm{T}}}(P, \lambda)$ for a matrix pencil or quadratic matrix polynomial $P(z)$ with $T$ palindromic structure. We also obtain the structured backward error for approximate real eigenvalues of real $T$-palindromic polynomials without restrictions on the degree of such polynomials.

Due to [1, Theorem 5.3.1], if $\lambda= \pm 1$, then there is no difference between the eigenvalue backward errors with respect to arbitrary perturbations and with respect to complex $T$-palindromic perturbations (when $P(z)$ is complex) and real $T$-palindromic perturbations (when $P(z)$ is real). This is proved for the weight vector $w=(1, \ldots, 1)$, but it may be easily generalized to arbitrary choices of palindromic weight vectors.

However, the situation is different if $\lambda \neq \pm 1$. Due to Theorem 3.2 the original Problem 2.3 of finding the structured backward error $\eta_{w}^{\text {palT }}(P, \lambda)$ for $T$-palindromic polynomials is equivalent to an optimization problem which requires maximizing the Rayleigh quotient of a Hermitian matrix subject to a number of constraints involving symmetric matrices. In these cases, the structured backward error may be obtained by using a theorem from [15]. To state this theorem and other results that follow from it, we recall that $\lambda_{2}(B)$ denotes the second largest eigenvalue of a Hermitian matrix $B$ and $\sigma_{2}(S)$ denotes the second largest singular value of a matrix $S$.

Theorem 5.1 (see [15]). Let $H \in \operatorname{Herm}(\mathrm{n})$ and $S \in \operatorname{Sym}(\mathrm{n})$ with $\operatorname{rank}(S) \geq 2$. Then

$$
\sup \left\{v^{*} H v \mid v \in \mathbb{C}^{n}, v^{T} S v=0,\|v\|=1\right\}=\min _{0 \leq t \leq t_{1}} \lambda_{2}\left(\left[\begin{array}{cc}
H & t \bar{S} \\
t S & \bar{H}
\end{array}\right]\right)
$$

where $t_{1}=\frac{2\|H\|}{\sigma_{2}(S)}$.

The following theorem gives a formula for the structured eigenvalue backward error $\eta_{w}^{\mathrm{pal}_{\mathrm{T}}}(P, \lambda)$ when $P(z)$ is a $T$-palindromic pencil and $\lambda \in \mathbb{C} \backslash\{0,1,-1\}$.

Theorem 5.2. Let $A \in \mathbb{C}^{n \times n}$ and suppose that $P(\lambda):=A+\lambda A^{T}$ is nonsingular for $\lambda \in \mathbb{C} \backslash\{0,1,-1\}$. Let $M:=\left(A+\lambda A^{T}\right)^{-1}$ and define $\gamma_{1}:=\sqrt{\frac{2}{1+|\lambda|}}, \gamma_{2}:=\sqrt{\frac{2|\lambda|}{1+|\lambda|}}$,

$$
\begin{gathered}
\widetilde{G}:=\left[\begin{array}{cc}
M^{*} M & \lambda M^{*} M \\
\bar{\lambda} M^{*} M & |\lambda|^{2} M^{*} M
\end{array}\right], C:=\left[\begin{array}{cc}
M^{T} & 0 \\
\lambda M^{T}-M & -\lambda M
\end{array}\right], \Gamma:=\left[\begin{array}{cc}
w_{0} \gamma_{1} I_{n} & 0 \\
0 & w_{0} \gamma_{2} I_{n}
\end{array}\right], \\
G:=\Gamma^{-1} \widetilde{G} \Gamma^{-1} \text { and } S:=\Gamma^{-1}\left(C+C^{T}\right) \Gamma^{-1} .
\end{gathered}
$$

Then

$$
\eta_{w}^{\mathrm{pal}_{\mathrm{T}}}(P, \lambda)=\left(\min _{0 \leq t \leq t_{1}} \lambda_{2}\left(\left[\begin{array}{cc}
G & t \bar{S} \\
t S & \bar{G}
\end{array}\right]\right)\right)^{-1 / 2}
$$

where $t_{1}=\frac{2\|G\|}{\sigma_{2}(S)}$.

Proof. Since $P(z)=A+z A^{T}, \quad(3.15)$ implies that

$$
\eta_{w}^{\mathrm{pal}_{\mathrm{T}}}(P, \lambda)=\left(\sup \left\{\frac{u^{*} G u}{u^{*} u} \mid u \in \mathbb{C}^{2 n} \backslash\{0\}, u^{T} S_{0} u=0,\right\}\right)^{-\frac{1}{2}},
$$

where $S_{0}=S$. The proof then follows by applying Theorem 5.1.

Similarly, the following theorem gives $\eta_{w}^{\text {pal }_{\mathrm{T}}}(P, \lambda)$ when $P(z)$ is a $T$-palindromic quadratic matrix polynomial and $\lambda \in \mathbb{C} \backslash\{0,1,-1\}$. 
Theorem 5.3. Let $P(z)=A_{0}+z A_{1}+z^{2} A_{0}^{T}$ be a T-palindromic quadratic polynomial and $\lambda \in \mathbb{C} \backslash\{0,1,-1\}$. Suppose that $\operatorname{det}(P(\lambda)) \neq 0$, and let $M:=(P(\lambda))^{-1}$. Furthermore, let $\gamma_{1}:=\sqrt{\frac{2}{1+|\lambda|^{2}}}$ and $\gamma_{2}:=\sqrt{\frac{2|\lambda|^{2}}{1+|\lambda|^{2}}}$, and define

$$
\begin{gathered}
\widetilde{G}:=\left[\begin{array}{ccc}
1 & \lambda & \lambda^{2} \\
\bar{\lambda} & |\lambda|^{2} & \lambda|\lambda|^{2} \\
\bar{\lambda}^{2} & \bar{\lambda}|\lambda|^{2} & |\lambda|^{4}
\end{array}\right] \otimes M^{*} M, \quad C:=\left[\begin{array}{ccc}
M^{T} & 0 & 0 \\
\lambda M^{T} & 0 & 0 \\
\lambda^{2} M^{T}-M & -\lambda M & -\lambda^{2} M
\end{array}\right], \\
\Gamma:=\operatorname{diag}\left(w_{0} \gamma_{1}, w_{1}, w_{0} \gamma_{2}\right) \otimes I_{n}, \quad G:=\Gamma^{-1} \widetilde{G} \Gamma^{-1} \text { and } S=\Gamma^{-1}\left(C+C^{T}\right) \Gamma^{-1} .
\end{gathered}
$$

Then

$$
\eta_{w}^{\mathrm{pal}_{\mathrm{T}}}(P, \lambda)=\left(\min _{0 \leq t \leq t_{1}} \lambda_{2}\left(\left[\begin{array}{cc}
G & t \bar{S} \\
t S & \bar{G}
\end{array}\right]\right)\right)^{-1 / 2}
$$

where $t_{1}=\frac{2\|G\|}{\sigma_{2}(S)}$.

Proof. Since $P(z)=A_{0}+z A_{1}+z^{2} A_{0}^{T}$, from (3.15) we have

$$
\eta_{w}^{\mathrm{pal}_{\mathrm{T}}}(P, \lambda)=\left(\sup \left\{\frac{u^{*} G u}{u^{*} u} \mid u \in \mathbb{C}^{3 n} \backslash\{0\}, u^{T} S_{0} u=0\right\}\right)^{-\frac{1}{2}}
$$

where $S_{0}=S$. The proof then follows by applying Theorem 5.1.

Remark 5.4. Due to (3.15), computing $\eta_{w}^{\mathrm{pal}_{\mathrm{T}}}(P, \lambda)$ for polynomials of degree greater than 2 involves maximizing the Rayleigh quotient $\frac{v^{*} G v}{v^{*} v}$ with respect to nonzero vectors $v$ that satisfy more than one constraint each involving a symmetric matrix. Generalizing our approach to compute $\eta_{w}^{\text {pal }}(P, \lambda)$ in these cases may involve obtaining appropriate extensions of Theorem 5.1. This does not seem to be straightforward and will be the subject of future research.

Remark 5.5. A strategy similar to the one outlined in Remark 4.4 may be used to compute $\eta_{w}^{\mathrm{pal}_{\mathrm{T}}}(P, \lambda)$ for the case that the $T$-palindromic perturbations affect only some of the coefficients of $P(z)$.

It may be noted that if $P(z)$ is a $T$-palindromic polynomials of even degree and only the coefficient $A_{\frac{m}{2}}$ is affected by perturbation, then there are no constraints in the computation of $\eta_{w}^{\text {pal }}(P, \lambda)$ and therefore it is equal to the backward error $\eta_{w}(P, \lambda)$ with respect to arbitrary perturbations. Moreover, $\eta_{w}^{\mathrm{pal}_{\mathrm{T}}}(P, \lambda)$ may be computed for any degree if the restrictions are such that the computation of the backward error involves only one constraint.

Remark 5.6. Note that in Theorem 3.2 if $\lambda \in \mathbb{R} \backslash\{0,1,-1\}$ and $P(z)$ is a real $T$-palindromic polynomial, then $G$ and $S_{i}$ are real Hermitian matrices. Therefore, denoting the real $\star$-palindromic structure by pal ${ }_{\star, \mathbb{R}}$, that is,

$$
\operatorname{pal}_{\star, \mathbb{R}}:=\left\{\left(\Delta_{0}, \ldots, \Delta_{m}\right) \in\left(\mathbb{R}^{n \times n}\right)^{m+1} \mid \Delta_{j}^{\star}=\Delta_{m-j}, j=0, \ldots, m\right\},
$$

we have

$$
\eta_{w}^{\mathrm{pal}_{T, \mathbb{R}}}(P, \lambda)=\eta_{w}^{\mathrm{pal}_{*, \mathbb{R}}}(P, \lambda)
$$

in such cases.

The final result of this section gives the structured backward error $\eta_{w}^{\mathrm{pal}_{T, \mathbb{R}}}(P, \lambda)$ when $\lambda \in \mathbb{R} \backslash\{0,1,-1\}$ and $P(z)$ is a real $T$-palindromic polynomial of any degree. 
It may be noted that despite the equality (5.1), this result is not a corollary of Theorem 4.2. However, the proof is based on similar arguments that make use of the real version of Theorem 4.1 with $\mathbb{C}$ replaced by $\mathbb{R}$ as given below.

Theorem 5.7. Let $G, H_{0}, \ldots, H_{p} \in \mathbb{R}^{n \times n}$ be Hermitian matrices. Assume that any nonzero linear combination $\alpha_{0} H_{0}+\cdots+\alpha_{p} H_{p},\left(\alpha_{0}, \ldots, \alpha_{p}\right) \in \mathbb{R}^{p+1} \backslash\{0\}$ is indefinite. (Here, "indefinite" is used in the same sense as in Theorem 4.1.) Then the following statements hold:

(1) The function $L: \mathbb{R}^{p+1} \rightarrow \mathbb{R},\left(t_{0}, \ldots, t_{p}\right) \mapsto \lambda_{\max }\left(G+t_{0} H_{0}+\cdots+t_{p} H_{p}\right)$ is convex and has a global minimum

$$
\lambda_{\max }^{\bullet}=\min _{t_{0}, \ldots, t_{p} \in \mathbb{R}} L\left(t_{0}, \ldots, t_{p}\right) .
$$

(2) If $p=0$ or the minimum $\lambda_{\max }^{\bullet}$ of $L$ is attained at $\left(\hat{t}_{0}, \ldots, \hat{t}_{p}\right) \in \mathbb{R}^{p+1}$ and is a simple eigenvalue of $H_{\bullet}:=G+\hat{t}_{0} H_{0}+\cdots+\hat{t}_{p} H_{p}$, then there exists an eigenvector $u \in \mathbb{R}^{n} \backslash\{0\}$ of $H_{\bullet}$ associated with $\lambda_{\max }^{\bullet}$ satisfying

$$
u^{T} H_{j} u=0 \quad \text { for } j=0, \ldots, p .
$$

(3) Under the assumptions of (2) we have

$$
\sup \left\{\frac{u^{T} G u}{u^{T} u} \mid u \neq 0, u^{T} H_{j} u=0, j=0, \ldots, p\right\}=\lambda_{\max }^{\bullet} .
$$

In particular, the supremum of the left-hand side of (5.3) is a maximum and attained for the eigenvector $u$ from (5.2).

Proof. The proofs of (1) and (3) follow from the proofs of the corresponding parts of $[6$, Theorem 3.2].

If $p=0$, then the proof of part (2) follows by arguing as in the proof of $[6$, Theorem 3.5] due to the fact that the set

$$
\left\{x^{T} H_{0} x \mid x \in \mathbb{R}^{n}, x^{T} x=1\right\}
$$

is convex. If $p>0$ and $\lambda_{\max }^{\bullet}$ is a simple eigenvalue of $H_{\bullet}$, then part (2) of Theorem 4.1 implies that there exists a nonzero (possibly complex) eigenvector $u$ corresponding to $\lambda_{\max }^{\bullet}$ of $H_{\bullet}$ that satisfies (5.2). However, as $H_{\bullet}$ is real and $\lambda_{\max }^{\bullet}$ is real and simple, the eigenvector $u$ can be chosen to be real. This proves part (2) and completes the proof of the theorem.

It is important to note that the assumption of simplicity of $\lambda_{\max }^{\bullet}$ made in the hypothesis of Theorem 5.7 is necessary even when $p=1$. This is evident from the following example, which is a slight modification of [6, Example 3.8].

Example 5.8. Let $G=\operatorname{diag}(\alpha, \alpha, \beta)$, where $\alpha>\beta \geq 0$. Also let

$$
H_{0}=\left[\begin{array}{ccc}
1 & 0 & 0 \\
0 & -1 & 0 \\
0 & 0 & 0
\end{array}\right] \text { and } H_{1}=\left[\begin{array}{ccc}
0 & 1 & 0 \\
1 & 0 & 0 \\
0 & 0 & 0
\end{array}\right] \text {. }
$$

Then any nonzero real linear combination of $H_{0}$ and $H_{1}$ is indefinite and for $t_{0}, t_{1} \in \mathbb{R}$, the matrix

$$
H\left(t_{0}, t_{1}\right)=G+t_{0} H_{0}+t_{1} H_{1}=\left[\begin{array}{ccc}
\alpha+t_{0} & t_{1} & 0 \\
t_{1} & \alpha-t_{0} & 0 \\
0 & 0 & \beta
\end{array}\right]
$$

Copyright (c) by SIAM. Unauthorized reproduction of this article is prohibited. 
has eigenvalues $\alpha \pm \sqrt{t_{0}^{2}+t_{1}^{2}}$ and $\beta$. Clearly the function $L: \mathbb{R}^{2} \rightarrow \mathbb{R}$ defined by $L\left(t_{0}, t_{1}\right)=\lambda_{\max }\left(H\left(t_{0}, t_{1}\right)\right)=\alpha+\sqrt{t_{0}^{2}+t_{1}^{2}}$ has its minimum $\lambda_{\max }^{\bullet}$ at $\left(t_{0}, t_{1}\right)=(0,0)$, i.e., when $H(0,0)=G$. But the maximal eigenvalue $\alpha$ of $G$ is a double eigenvalue with corresponding eigenvectors $e_{1}$ and $e_{2}$ which are the first two basis vectors of $\mathbb{R}^{3}$. Therefore, the matrix whose columns form an orthonormal basis of the eigenspace of $G$ corresponding to $\alpha$ is $U=\left[\begin{array}{ll}e_{1} & e_{2}\end{array}\right] \in \mathbb{R}^{3 \times 2}$. There exists a real nonzero vector $x$ in the eigenspace of $G$ corresponding to $\alpha$ satisfying $x^{*} H_{0} x=x^{*} H_{1} x=0$ if and only if the real joint numerical range of the matrices

$$
U_{1}:=U^{T} H_{0} U=\left[\begin{array}{cc}
1 & 0 \\
0 & -1
\end{array}\right] \text { and } U_{2}:=U^{T} H_{1} U=\left[\begin{array}{cc}
0 & 1 \\
1 & 0
\end{array}\right]
$$

defined by

$$
\left\{\left(x^{T} U_{1} x, x^{T} U_{2} x\right) \mid x \in \mathbb{R}^{2},\|x\|_{2}=1\right\}
$$

contains 0 . Clearly this is not true in this case as this set represents the unit circle.

Note that this example does not contradict Theorem 4.1 as the eigenvector of $G$ with respect to $\alpha$ that satisfies $u^{*} H_{0} u=u^{*} H_{1} u=0$ is the complex vector $u=\left[\begin{array}{ll}1 & i\end{array}\right]^{T}$.

The following theorem gives formulae for the structured eigenvalue backward error $\eta_{w}^{\mathrm{pal}_{\mathrm{T}}}(P, \lambda)$ when $P(z)$ is a real $T$-palindromic polynomial and $\lambda \in \mathbb{R} \backslash\{0, \pm 1\}$.

THEOREM 5.9. Let $P(z)=\sum_{j=0}^{m} z^{j} A_{j}$ be a real T-palindromic polynomial and $\lambda \in \mathbb{R} \backslash\{0, \pm 1\}$. Suppose that $\operatorname{det}(P(\lambda)) \neq 0$ so that $M=(P(\lambda))^{-1}$ exists and set $k=\left\lfloor\frac{m-1}{2}\right\rfloor$. Then

$$
\lambda_{\max }^{\bullet}:=\min _{t_{0}, \ldots, t_{k} \in \mathbb{R}} \lambda_{\max }\left(G+t_{0} S_{0}+\cdots+t_{k} S_{k}\right)
$$

is attained for some $\left(\hat{t}_{0}, \ldots, \hat{t}_{k}\right) \in \mathbb{R}^{k+1}$, where $G$ is defined by (3.12) and $S_{j}, j=$ $0, \ldots, k$, are defined by (3.14), respectively. If $m=1$ or $m=2$, or if $\lambda_{\max }^{\bullet}$ is a simple eigenvalue of $G+\hat{t}_{0} S_{0}+\cdots+\hat{t}_{k} S_{k}$, then

$$
\eta_{w}^{\mathrm{pal}_{\mathrm{T}, \mathbb{R}}}(P, \lambda)=\frac{1}{\sqrt{\lambda_{\max }^{\bullet}}}=\left(\min _{t_{0}, \ldots, t_{k} \in \mathbb{R}} \lambda_{\max }\left(G+t_{0} S_{0}+\cdots+t_{k} S_{k}\right)\right)^{-1 / 2} .
$$

Proof. Consider the Hermitian matrices $\widetilde{S}_{j}:=\widetilde{C}_{j}+\widetilde{C}_{j}^{T}, j=0, \ldots, k$, where $\widetilde{C}_{j}$ are as defined in (3.5). Observe that $k=0$ whenever $m=1$ or $m=2$. Therefore, the proof follows from Theorem 5.7 if it is established that each nontrivial linear combination of $S_{0}, \ldots, S_{k}$ given by (3.14) or, equivalently, of $\widetilde{S}_{0}, \ldots, \widetilde{S}_{k}$ is indefinite. Suppose there exists $\left[\alpha_{0}, \ldots, \alpha_{k}\right]^{T} \in \mathbb{R}^{k+1}$ such that $S:=\sum_{j=0}^{k} \alpha_{j} \widetilde{S}_{j}$ is semidefinite. Then recalling that $\Lambda_{m}=\left[1, \lambda, \ldots, \lambda^{m}\right] \in \mathbb{C}^{1 \times(m+1)}$, we have

$$
\begin{aligned}
S & =\sum_{j=0}^{k} \alpha_{j}\left(\left(\Lambda_{m}^{T}\left(e_{j+1}^{T}-e_{m+1-j}^{T}\right)\right) \otimes M^{T}+\left(\left(e_{j+1}-e_{m+1-j}\right) \Lambda_{m}\right) \otimes M\right) \\
& =\left(\Lambda_{m}^{T} \alpha^{T}\right) \otimes M^{T}+\left(\alpha \Lambda_{m}\right) \otimes M,
\end{aligned}
$$

where the vector $\alpha$ is given by $\alpha:=\left[\alpha_{0}, \ldots, \alpha_{k},-\alpha_{k}, \ldots,-\alpha_{0}\right]^{T}$ when $m$ is odd and by $\alpha:=\left[\alpha_{0}, \ldots, \alpha_{k}, 0,-\alpha_{k}, \ldots,-\alpha_{0}\right]^{T}$ when $m$ is even. 
Setting $Q$ as in (4.3) and $a=\left[a_{0}, \ldots, a_{m}\right]^{T}:=Q^{T} \alpha$, since $\Lambda_{m} Q=e_{1}^{T}$, we have

$$
\begin{aligned}
\left(Q \otimes I_{n}\right)^{T} S\left(Q \otimes I_{n}\right) & =\left(Q^{T} \Lambda_{m}^{T} \alpha^{T} Q\right) \otimes M^{T}+\left(Q^{T} \alpha \Lambda_{m} Q_{m}\right) \otimes M \\
& =\left(e_{1} a^{T}\right) \otimes M^{T}+\left(a e_{1}^{T}\right) \otimes M \\
& =\left[\begin{array}{cccc}
a_{0}\left(M+M^{T}\right) & a_{1} M^{T} & \cdots & a_{m} M^{T} \\
a_{1} M & 0 & \cdots & 0 \\
\vdots & \vdots & & \vdots \\
a_{m} M & 0 & \cdots & 0
\end{array}\right] .
\end{aligned}
$$

If $S$ is semidefinite, then $a_{1}=\cdots=a_{m}=0$, which implies that $Q^{T} \alpha=a=a_{0} e_{1}$. Therefore, $a_{0}=\alpha_{0}$ and

$$
-\lambda \alpha_{j-1}+\alpha_{j}=0, j=1, \ldots, k .
$$

Also,

$$
\begin{aligned}
(\lambda+1) \alpha_{k} & =0 \text { when } m \text { is odd and } \\
\lambda \alpha_{k} & =0 \text { when } m \text { is even. }
\end{aligned}
$$

The identities (5.4) imply that

$$
\alpha_{k}=\lambda^{k} \alpha_{0} .
$$

When $m$ is odd, we have $\alpha_{k}=0$ from (5.5) since $\lambda \neq-1$. Similarly when $m$ is even, (5.6) gives $\alpha_{k}=0$ as $\lambda \neq 0$. In either case, (5.7) implies that $a_{0}=\alpha_{0}=0$ as $\lambda \neq 0$ and completes the proof.

Remark 5.10. A strategy identical to the one suggested in Remark 4.5 gives an optimal $T$-palindromic perturbation to $P(z)$ corresponding to $\eta_{w}^{\mathrm{pal}_{\mathrm{T}}}(P, \lambda)$ in Theorems 5.2 and 5.3 and an optimal real $T$-palindromic perturbation to $P(z)$ corresponding to $\eta_{w}^{\mathrm{pal}_{\mathrm{T}, \mathbb{R}}}(P, \lambda)$ in Theorem 5.9.

6. Numerical experiments. In this section we present some numerical examples to illustrate the proposed method for computing the structured backward error $\eta_{w}^{\mathbb{S}}(P, \lambda)$ of some $\lambda \in \mathbb{C} \backslash\{0\}$ for the structures $\mathbb{S}=$ pal $_{\star}$ and for $w=(1,1, \ldots, 1)$. In all cases we have used the software package CVX [8, 9] in MATLAB to solve the associated optimization problems.

Example 6.1. $L(z)=A+z A^{*}$ is a $*$-palindromic pencil of size 4 with eigenvalues $0.4624-0.8867 i,-0.5697+1.7298 i,-0.1718+0.5215 i,-0.9765+0.2155 i$. For $\lambda=0.4853-0.5955 i$ the backward error with respect to arbitrary perturbations is $\eta_{w}(L, \lambda)=0.0912$, while the structured backward error satisfies $\eta_{w}^{\mathrm{pal}_{*}}(L, \lambda)=0.3320$. The plot on the left of Figure 1 illustrates the movement of the eigenvalues of the pencil $L(z)$ under the homotopic perturbations $L(z)+t \Delta L(z)$ as $t$ varies from 0 to 1 and $\Delta L(z)$ is an optimal $*$-palindromic perturbation corresponding to $\eta_{w}^{\mathrm{pal}_{*}}(L, \lambda)$ that induces eigenvalues at $(\lambda, 1 / \bar{\lambda})$. The eigenvalue curves starting from $0.4624-0.8867 i$ and $-0.9765+0.2155 i$ (each marked by a star surrounded by a circle) come together on the unit circle and split out to form the pair of eigenvalues $(\lambda, 1 / \bar{\lambda}$ ) (where $\lambda$ is marked by a star surrounded by a diamond) of the pencil $L(z)+\Delta L(z)$.

On the other hand, the plot on the right-hand side of Figure 1 gives the movement of the eigenvalues under the homotopic perturbations $L(z)+t \widetilde{\Delta L}(z)$ when $t$ moves from 0 to 1 and $\widetilde{\Delta L}(z)$ is an optimal perturbation corresponding to $\eta_{w}(L, \lambda)$ that 

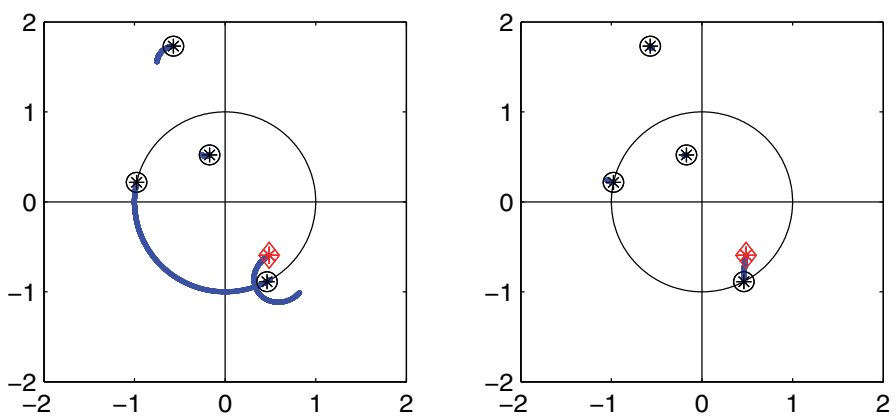

FIG. 1. Eigenvalue perturbation curves for the *-palindromic pencil $L(z)$ of Example 6.1 with respect $*$-palindromic perturbations (left) and arbitrary perturbations (right).

induces an eigenvalue at $\lambda$ without preserving $*$-palindromic structure. In this case the perturbations move the nearest eigenvalue of the pencil to $\lambda$.

Example 6.2. $Q(z)=A+z B^{*}+z^{2} A^{*}$ is a $*$-palindromic polynomial of size 3 with eigenvalues $-3.2746-0.4165 i,-0.3597+1.82221 i, 0.9896-0.1437 i,-0.0961-$ $0.9954 i,-0.3005-0.0382 i,-0.1043+0.5282 i$. For $\lambda=0.88+0.15 i$, the eigenvalue backward error with respect to arbitrary perturbations is 0.609 , while with respect to $*$-palindromic perturbations this is 1.7059 . The plot on the left of Figure 2 illustrates the effect of perturbations $Q(z)+t \Delta Q(z)$ on the eigenvalues of $Q(z)$ as $t$ varies from 0 to $1, \Delta Q(z)$ being an optimal $*$-palindromic perturbation to $Q(z)$ corresponding to $\eta_{w}^{\mathrm{pal}_{*}}(Q, \lambda)$ that induces eigenvalues at $(\lambda, 1 / \bar{\lambda})$. It shows eigenvalue curves starting from the eigenvalues $-0.1043+0.528 i$ and $-0.3597+1.82221 i$ (each marked by a star surrounded by circle) of $Q(z)$ coalescing on the unit circle and moving along the circle till they next coalesce with the eigenvalue curve starting from the eigenvalue $0.9896-0.1437 i$ on the unit circle. After the second coalescence, the eigenvalue curves split out of the unit circle to form the pair of eigenvalues $(\lambda, 1 / \bar{\lambda}$ ) (where $\lambda$ is marked by a star surrounded by a diamond) of $Q(z)+\Delta Q(z)$.

The plot on the right of Figure 2 shows the movement of the eigenvalues of $Q(z)$ under perturbations $Q(z)+t \widetilde{\Delta Q}(z)$, where $\widetilde{\Delta Q}(z)$ is an optimal perturbation to $Q(z)$ corresponding to $\eta_{w}(Q, \lambda)$ that induces an eigenvalue at $\lambda$ and is not $*$-palindromic. In this case the nearest eigenvalue of $Q(z)$ on the unit circle moves to form the eigenvalue $\lambda$ of $Q(z)+\widetilde{\Delta Q}(z)$.

Example 6.3. $L(z)=A+z A^{T}$ is a real $T$-palindromic pencil of size 3 with eigenvalues -1 and $-0.5954 \pm 0.8034 i$ all on the unit circle. For $\lambda=-1.6656$, the eigenvalue backward error is 0.6563 with respect to real $T$-palindromic perturbations and 0.5614 with respect to complex $T$-palindromic perturbations. With respect to arbitrary perturbations, the eigenvalue backward error is 0.3177 . Figure 3 illustrates the effect of real $T$-palindromic, complex $T$-palindromic, and arbitrary perturbations on the eigenvalues of $L(z)$ so that they move to form an eigenvalue at $\lambda$ for the respective perturbed pencils.

The plot on the left of Figure 3 shows the effect of perturbations $L(z)+t \Delta L(z)$ on the eigenvalues of $L(z)$ (in thick curves) as $t$ moves from 0 to $1, \Delta L(z)$ being the minimal real $T$-palindromic perturbation to $L(z)$ corresponding to $\eta_{w}^{\mathrm{pal}_{T, \mathbb{R}}}(L, \lambda)$ that induces eigenvalues at $(\lambda, 1 / \lambda)$. In this case eigenvalue curves starting from the eigenvalues $-0.5954 \pm 0.8034 i$ (each marked by a star surrounded by a circle) on the 

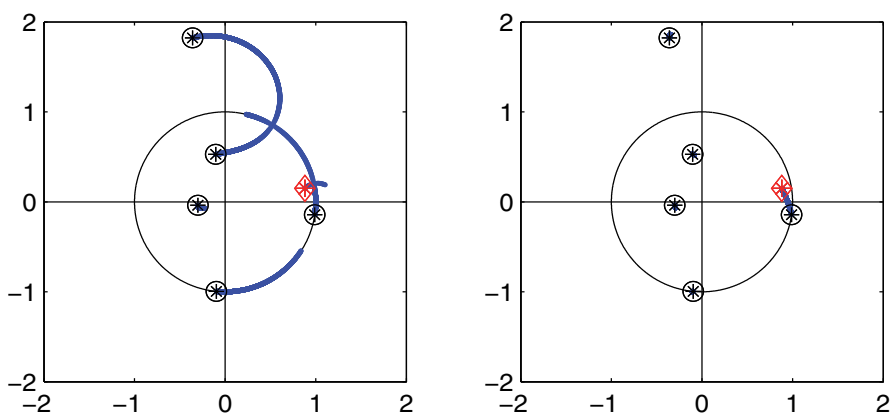

FIG. 2. Eigenvalue perturbation curves for the *-palindromic polynomial $Q(z)$ of Example 6.2 with respect to $*$-palindromic perturbations (left) and arbitrary perturbations (right).
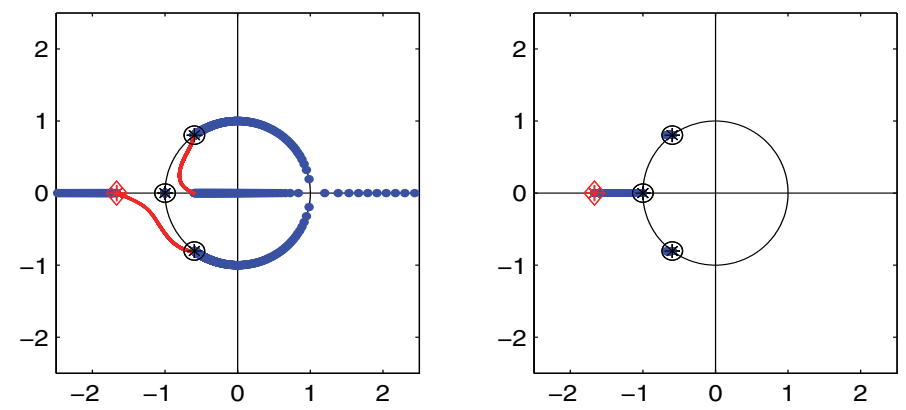

FIG. 3. Eigenvalue perturbation curves for the real T-palindromic pencil $L(z)$ of Example 6.3 with respect to real and complex $T$-palindromic perturbations (left) and arbitrary perturbations (right).

unit circle coalesce on the unit circle and split out with one of the branches moving over $\infty$ to form the eigenvalue $\lambda$ (marked by a star surrounded by a diamond) and the other moving out to form the eigenvalue $1 / \lambda$ (inside the unit circle) as $t$ moves from 0 to 1 .

The movement of the perturbed eigenvalues under the given real $T$-palindromic perturbations may partly be attributed to two facts. The first is that -1 is always an eigenvalue of a $T$-palindromic polynomial of odd degree and odd size (since $P(-1)$ is then a skew symmetric matrix of odd size and thus singular) of which the given pencil $L(z)$ is a particular case. The second fact is that eigenvalues of real $T$-palindromic polynomials occur in quadruples $(\mu, \bar{\mu}, 1 / \mu, 1 / \bar{\mu})$. This symmetry breaks down only on the unit circle and on the real line where it reduces to the pairing $(\mu, 1 / \mu)$. Since the only eigenvalues of $L(z)$ other than -1 are also on the unit circle, in order to maintain the eigenvalue symmetry, they have to pass through the intersection of the unit circle and the real line to form the eigenvalues at $\lambda$ and $1 / \lambda$.

The plot on the left of Figure 3 also shows the effect of perturbations $L(z)+t \widehat{\Delta L}(z)$ on the eigenvalues of $L(z)$ (in thin curves) as $t$ moves from 0 to $1, \widehat{\Delta L}(z)$ being the minimal complex $T$-palindromic perturbation to $L(z)$ corresponding to $\eta_{w}^{\mathrm{pal}_{\mathrm{T}}}(L, \lambda)$ that induces eigenvalues at $(\lambda, 1 / \lambda)$. Also in this case -1 cannot be moved to $\lambda$ under $T$-palindromic perturbations. Instead, an eigenvalue curve starting from $-0.5954+$ $0.8034 i$ moves to $\lambda$ while another starting from $-0.5954-0.8034 i$ moves to $1 / \lambda$ (inside the unit circle) as $t$ moves from 0 to 1 . 
TABLE 1

Values of $\eta_{w}(L, \lambda)$ and $\eta_{w}^{\mathrm{pal}_{*}}(L, \lambda)$ for the *-palindromic pencil $L(z)$ in Example 6.1, where $\lambda \rightarrow 0.4624-0.8867 i$.

\begin{tabular}{|c|c|c|}
\hline$\lambda$ & $\eta_{w}(L, \lambda)$ & $\eta_{w}^{\mathrm{pa}}{ }_{*}(L, \lambda)$ \\
\hline $0.600-1.200 \mathrm{i}$ & 0.0710 & 0.2755 \\
\hline $0.550-1.100 \mathrm{i}$ & 0.0510 & 0.2772 \\
\hline $0.520-1.000 \mathrm{i}$ & 0.0300 & 0.2824 \\
\hline $0.500-0.980 \mathrm{i}$ & 0.0240 & 0.2807 \\
\hline $0.480-0.950 \mathrm{i}$ & 0.0159 & 0.2799 \\
\hline $0.475-0.930 \mathrm{i}$ & 0.0111 & 0.2811 \\
\hline $0.470-0.900 \mathrm{i}$ & 0.0038 & 0.2835 \\
\hline $0.465-0.895 \mathrm{i}$ & 0.0022 & 0.2830 \\
\hline
\end{tabular}

TABLE 2

Values of $\eta_{w}(L, \lambda)$ and $\eta_{w}^{\mathrm{pal}_{*}}(L, \lambda)$ for the *-palindromic pencil $L(z)$ in Example 6.1, where $\lambda \rightarrow-0.5697+1.7298 i$ (left) and for arbitrary $\lambda$ (right).

\begin{tabular}{|c|c|c|}
\hline$\lambda$ & $\eta_{w}(L, \lambda)$ & $\eta_{w}^{\mathrm{pa}_{*}}(L, \lambda)$ \\
\hline$-2.50+0.50 i$ & 0.1134 & 0.1605 \\
\hline$-2.00+1.00 i$ & 0.1006 & 0.1344 \\
\hline$-1.50+1.40 i$ & 0.0882 & 0.1012 \\
\hline$-0.90+1.50 i$ & 0.0569 & 0.0593 \\
\hline$-0.60+1.62 i$ & 0.0196 & 0.0203 \\
\hline$-0.58+1.70 i$ & 0.0053 & 0.0055 \\
\hline
\end{tabular}

\begin{tabular}{|c|c|c|}
\hline$\lambda$ & $\eta_{w}(L, \lambda)$ & $\eta_{w}^{\mathrm{pal}_{*}}(L, \lambda)$ \\
\hline $1.1890+0.0376 i$ & 0.4726 & 0.5937 \\
\hline $0.2940-1.3362 i$ & 0.0850 & 0.2190 \\
\hline $1.1910-1.2025 i$ & 0.1571 & 0.3415 \\
\hline $0.9410-0.9921 i$ & 0.1173 & 0.3494 \\
\hline $0.4850-0.5955 i$ & 0.0912 & 0.3320 \\
\hline $0.6680-0.0783 i$ & 0.3688 & 0.5149 \\
\hline
\end{tabular}

Values of $\eta_{w}(L, \lambda), \eta_{w}^{\mathrm{pal}_{\mathrm{T}}}(L, \lambda)$ and $\eta_{w}^{\text {PaBLE }, \mathbb{R}}(L, \lambda)$ for the $T$-palindromic pencil $L(z)$ in Example 6.3 as $\lambda \rightarrow-1$.

\begin{tabular}{|c|c|c|c|}
\hline$\lambda$ & $\eta_{w}(P, \lambda)$ & $\eta_{w}^{\mathrm{pal}_{\mathrm{T}}}(P, \lambda)$ & $\eta_{w}^{\mathrm{pal}_{\mathrm{T}, \mathrm{R}}}(P, \lambda)$ \\
\hline-1.6656 & 0.1692 & 0.3177 & 0.5614 \\
\hline-1.5500 & 0.1501 & 0.3076 & 0.5623 \\
\hline-1.4500 & 0.1308 & 0.2992 & 0.5631 \\
\hline-1.3500 & 0.1086 & 0.2912 & 0.5638 \\
\hline-1.2500 & 0.0827 & 0.2842 & 0.5644 \\
\hline-1.1500 & 0.0528 & 0.2788 & 0.5649 \\
\hline
\end{tabular}

Finally, the plot on the right of Figure 3 shows the effect of perturbations $L(z)+$ $\widetilde{\Delta L}(z)$ on the eigenvalues of $L(z)$ as $t$ moves from 0 to $1, \widetilde{\Delta L}(z)$ being an optimal perturbation corresponding to $\eta_{w}(L, \lambda)$ that induces an eigenvalue at $\lambda$ and is not $T$-palindromic. In this case, the nearest eigenvalue -1 of $L(z)$ moves to form the eigenvalue $\lambda$ of $L(z)+\widetilde{\Delta L}(z)$.

We also compare $\eta_{w}(P, \lambda)$ with $\eta_{w}^{\mathrm{pal}_{\star}}(P, \lambda)$ for the cases that the values of $\lambda$ converge to an eigenvalue of $P(z)$ as well as for arbitrary values of $\lambda$.

Table 1 illustrates these comparisons for the $*$-palindromic pencil $L(z)$ of Example 6.1 as $\lambda$ values converge to the eigenvalue $0.4624-0.8867 i$ on the unit circle. Observe that while $\eta_{w}(L, \lambda)$ decreases to 0 , this is not the case for $\eta_{w}^{\mathrm{pal}_{*}}(L, \lambda)$, leading to large differences in the values of the two backward errors.

Table 2 does the same comparison for values of $\lambda$ that converge to the eigenvalue $-0.5697+1.7298 i$ not on the unit circle as well as for arbitrary values of $\lambda$. In the first case, both $\eta_{w}(L, \lambda)$ and $\eta_{w}^{\mathrm{pal}_{*}}(L, \lambda)$ decrease to 0 . However, in the second case, there is a significant difference between the two backward errors even when the values of $\lambda$ are away from the unit circle.

Table 3 compares the backward errors $\eta_{w}(L, \lambda), \eta_{w}^{\mathrm{pal}_{\mathrm{T}, \mathbb{R}}}(L, \lambda)$ and $\eta_{w}^{\mathrm{pal}_{\mathrm{T}}}(L, \lambda)$ for the $T$-palindromic pencil $L(z)$ in Example 6.3 as $\lambda$ converges to -1 along the real line. 
Observe that while both $\eta_{w}(L, \lambda)$ and $\eta_{w}^{\mathrm{pal}_{\mathrm{T}}}(L, \lambda)$ decrease, $\eta_{w}^{\mathrm{pal}_{\mathrm{T}, \mathbb{R}}}(L, \lambda)$ increases as $\lambda$ approaches -1 . This leads to large differences between $\eta_{w}^{\text {pal }{ }_{\mathrm{T}, \mathbb{R}}}(L, \lambda)$ and the other backward errors at values of $\lambda$ close to -1 .

Conclusions. We have obtained formulas for the backward error of approximate eigenvalues of $*$-palindromic matrix polynomials and $T$-palindromic pencils and quadratic polynomials with respect to structure preserving perturbations. When the $T$-palindromic polynomial is real, we have also obtained the backward error of a real number considered as an approximate eigenvalue of the matrix polynomial with respect to real $T$-palindromic perturbations. For each case, a procedure for constructing an optimal perturbation that corresponds to the structured backward error is provided. Numerical experiments suggest that there is a significant difference between the backward errors with respect to structure preserving and arbitrary perturbations in many cases.

\section{REFERENCES}

[1] B. Adhikari, Backward Perturbation and Sensitivity Analysis of Structured Polynomial Eigenvalue Problems, Ph.D. thesis, Department of Mathematics, Indian Institute of Technology Guwahati, 2008.

[2] B. Adhikari And R. Alam, Structured backward errors and pseudospectra of structured matrix pencils, SIAM J. Matrix Anal. Appl., 31 (2009), pp. 331-359.

[3] B. Adhikari And R. Alam, On backward errors of structured polynomial eigenproblems solved by structure preserving linearizations, Linear Algebra Appl., 434 (2011), pp. 1989-2017.

[4] Sk. S. Ahmad and R. Alam, Pseudospectra, critical points and multiple eigenvalues of matrix polynomials, Linear Algebra Appl., 430 (2009), pp. 1171-1195.

[5] R. Alam, S. Bora, M Karow, V. Mehrmann, and J. Moro, Perturbation theory for Hamiltonian matrices and the distance to bounded-realness, SIAM J. Matrix Anal. Appl. 32 (2011), pp. 484-514.

[6] S. Bora, M. Karow, C. Mehl, And P. Sharma, Structured eigenvalue backward errors of matrix pencils and polynomials with Hermitian and related structures, SIAM J. Matrix Anal. Appl., 35 (2014), pp. 453-475.

[7] I. Gohberg, P. Lancaster, and L. Rodman, Indefinite Linear Algebra, Birkhäuser, Basel, 2005.

[8] M. Grant And S. Boyd, Graph implementations for nonsmooth convex programs, in Recent Advances in Learning and Control (A Tribute to M. Vidyasagar), V. Blondel, S. Boyd, and H. Kimura, eds., pp. 95-110; Lecture Notes in Control and Inform. Sci. 371, Springer, New York, 2008, also available online from http://stanford.edu/ boyd/graph_dcp.html.

[9] M. Grant and S. Boyd, CVX: MATLAB Software for Disciplined Convex Programming, Version 2.0 Beta, http://cvxr.com/cvx (2012).

[10] D. J. Higham and N. J. Higham, Structured backward error and condition of generalized eigenvalue problems, SIAM J. Matrix Anal. Appl., 20 (1998), pp. 493-512.

[11] R. A. Horn and C. R. Johnson, Topics in Matrix Analysis, Cambridge University Press, Cambridge, UK, 1991.

[12] A. Hilliges, Numerische Lösung von quadratischen Eigenwertproblemen mit Anwendung in der Schienendynamik, Diplomarbeit, Institut für Mathematik, TU Berlin, 2004.

[13] A. Hilliges, C. Mehl, and V. Mehrmann, On the solution of palindromic eigenvalue problems, in Proceedings of the 4th European Congress on Computational Methods in Applied Sciences and Engineering (ECCOMAS), Jyväskylä, Finland, 2004.

[14] W. Kahan, B. N. Parlett, And E. JiAng, Residual bounds on approximate eigensystems of nonnormal matrices, SIAM J. Numer. Anal., 19 (1982), pp. 470-484.

[15] M. KAROW, $\mu$-values and spectral value sets for linear perturbation classes defined by a scalar product, SIAM J. Matrix Anal. Appl., 32 (2011), pp. 845-865.

[16] M. Karow, D. Kressner, And F. Tisseur, Structured eigenvalue condition numbers, SIAM J. Matrix Anal. Appl., 28 (2006), pp. 1052-1068.

[17] C. G. Khatri And S. K. Mitra, Hermitian and nonnegative definite solutions of linear matrix equations, SIAM J. Appl. Math., 31 (1976), pp. 579-585.

Copyright $@$ by SIAM. Unauthorized reproduction of this article is prohibited. 
[18] D. Kressner, M. J. Peláez, And J. Moro, Structured Hölder condition numbers for multiple eigenvalues, SIAM J. Matrix Anal. Appl., 31 (2009), pp. 175-201.

[19] D. Kressner, C. Schröder and D. S. Watkins, Implicit $Q R$ algorithms for palindromic and even eigenvalue problems, Numer. Algorithms, 51 (2009), pp. 209-238.

[20] P. Lancaster, Lambda-Matrices and Vibrating Systems, Pergamon Press, Oxford, UK, 1966.

[21] P. Lancaster and L. Rodman, Algebraic Riccati Equations, Clarendon Press, Oxford, UK, 1995.

[22] D. S. Mackey, N. Mackey, C. Mehl, and V. Mehrmann, Palindromic polynomial eigenvalue problems: Good vibrations from good linearizations, SIAM J. Matrix Anal. Appl., 28 (2006), pp. 1029-1051.

[23] D. S. Mackey, N. Mackey, C. Mehl, and V. Mehrmann, Numerical methods for palindromic eigenvalue problems: Computing the anti-triangular Schur form, Numer. Linear Algebra Appl., 16 (2009), pp. 63-86.

[24] D. S. Mackey, N. Mackey, And F. Tisseur, Structured mapping problems for matrices associated with scalar products Part I: Lie and Jordan algebras, SIAM J. Matrix Anal. Appl., 29 (2008), pp. 1389-1410.

[25] V. Mehrmann, The Autonomous Linear Quadratic Control Problem. Theory and Numerical Solution, Lecture Notes in Control and Inform. Sci. 163, Springer-Verlag, Berlin, 1991.

[26] F. Tisseur, Backward error and condition of polynomial eigenvalue problems, Linear Algebra Appl., 309 (2000), pp. 339-361.

[27] F. Tisseur, A chart of backward errors and condition numbers for singly and doubly structured eigenvalue problems, SIAM J. Matrix Anal. Appl., 24 (2003), pp. 877-897.

[28] F. Tisseur and K. Meerbergen, The quadratic eigenvalue problem, SIAM Rev., 43 (2001), pp. 235-286.

[29] S. Zaglmayr, Eigenvalue Problems in SAW-Filter Simulations, Diplomarbeit, Johannes Kepler University Linz, Austria, 2002.

Copyright (c) by SIAM. Unauthorized reproduction of this article is prohibited. 